UNIVERSITY OF GOTHENBURG

SCHOOL OF BUSINESS, ECONOMICS AND LAW

WORKING PAPERS IN ECONOMICS

No 502

\title{
Dynamic Hedging of Portfolio Credit Risk in a Markov Copula Model
}

Tomasz R. Bielecki, Areski Cousin, Stéphane Crépey and Alexander Herbertsson

Revised: October 2012

ISSN 1403-2473 (print)

ISSN 1403-2465 (online) 


\title{
Dynamic Hedging of Portfolio Credit Risk in a Markov Copula Model
}

\author{
Tomasz R. Bielecki ${ }^{1, *}$, Areski Cousin ${ }^{2 \dagger}$, \\ Stéphane Crépey ${ }^{3, \ddagger}, \quad$ Alexander Herbertsson ${ }^{4, \S}$ \\ ${ }^{1}$ Department of Applied Mathematics \\ Illinois Institute of Technology, Chicago, IL 60616, USA \\ ${ }^{2}$ Université de Lyon, Université Lyon 1, LSAF, France \\ ${ }^{3}$ Laboratoire Analyse et Probabilités \\ Université d'Évry Val d'Essonne, 91025 Évry Cedex, France \\ ${ }^{4}$ Centre for finance/Department of Economics \\ University of Gothenburg, SE 40530 Göteborg, Sweden
}

October 8, 2012

\begin{abstract}
We consider a bottom-up Markovian copula model of portfolio credit risk where dependence among credit names mainly stems from the possibility of simultaneous defaults. Due to the Markovian copula nature of the model, calibration of marginals and dependence parameters can be performed separately using a two-steps procedure, much like in a standard static copula set-up. In addition, the model admits a common shocks interpretation, which is a very important feature as, thanks to it, efficient convolution recursion procedures are available for pricing and hedging CDO tranches, conditionally on any given state of the underlying multivariate Markov process. As a result this model allows us to dynamically hedge CDO tranches using single-name CDSs in a theoretically sound and practically convenient way. To illustrate this we calibrate the model against market data on CDO tranches and the underlying single-name CDSs. We then study the loss distributions as well as the min-variance hedging strategies in the calibrated portfolios.
\end{abstract}

Keywords: Portfolio Credit Risk, Basket Credit Derivatives, Markov Copula Model, Common Shocks, Dynamic Min-Variance Hedging.

\footnotetext{
${ }^{*}$ The research of T.R. Bielecki was supported by NSF Grant DMS-0604789 and NSF Grant DMS-0908099.

${ }^{\dagger}$ The research of A. Cousin benefited from the support of the DGE and the ANR project Ast\&Risk.

${ }^{\ddagger}$ The research of S. Crépey benefited from the support of the 'Chaire Risque de crédit', Fédération Bancaire Française.

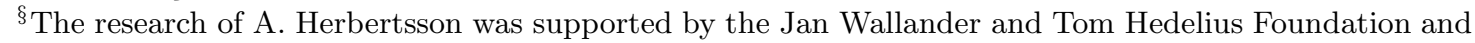
by Vinnova
} 


\section{Introduction}

The CDO market have been deeply and adversely impacted by the last financial crises. In particular, CDO issuances have become quite rare. Nevertheless, there are huge notionals of CDO contracts outstanding and market participants continue to be confronted with the task to hedge their positions in these contracts up to maturity date. Moreover, according to the current regulation (see [3]), tranches on standard indices and their associated liquid hedging positions continue to be charged as hedge-sets under internal VaR-based method, which makes the issue of hedging even more important for standardized CDO tranches. For previous studies of this issue we refer the reader to, among others, Laurent, Cousin and Fermanian [31, Frey and Backhaus [24], Cont and Kan [15] or Cousin, Crépey and Kan [17]. In particular it has been established empirically in [15] and 17 that a single-instrument hedge of a CDO tranche by the corresponding credit index is often not good enough. In this paper we deal with a bottom-up Markovian copula model, in which hedging loss derivatives by single-name instruments can be performed in a theoretically sound and practical way.

There are two major theoretical contributions of the paper:

- First, we construct a Markov copula model that is adequate for the problem at hand, that is for the problem of dynamic hedging of portfolio credit risk. The (dynamic) copula property of the model allows for separation of calibration of the univariate marginals of the underlying multivariate Markov process, from calibration of the dependence structure between the components of the process. This is of critical importance from the practical point of view.

- Second, we provide the common shocks interpretation of our Markovian copula model. This is important from the practical point of view as this interpretation underlies semiexplicit, convolution based pricing and Greeking schemes for basket credit derivatives. Such numerical schemes play a crucial role when calibrating credit portfolio models and in related applications such as counterparty risk valuation for portfolios (see [2, 6]). This allows one to address in a dynamic and theoretically consistent way the issues of hedging basket credit derivatives by individual names, whilst preserving the static common factor tractability.

The common shock aspect of our model is related to the work by Elouerkhaoui 22 . (see also Brigo et al. [12, 13, 14]). Consequently, some results derived in this paper are consistent with results derived in [22]. However, there are major differences between our study and the one presented in [22]:

- First of all, while Elouerkhaoui [22] works in a point-process set-up, we use a Markovian model; the practical interest of our framework is thus an increased model tractability, especially with regard to the dynamic hedging aspect of our approach;

- In particular, the approach of [22] suffers from the "curse of dimensionality" due to the need of summation (integration) over the set denoted by $\Pi_{n}$ in [22] (see for example equation (2.6) therein, and compare with our own result (17) below), the set of all subsets of the set $\{1,2, \ldots, n\}$. By contrast, the complexity of our formula (7) for the generator of our Markov process, or of our common shock algorithms described in Subsection 3.3, are controlled by the cardinality of our triggering-events set $\mathcal{Y}$, which one can typically be taken of the order of $n$ in applications; see Section 4 . 
- Secondly, as already stated, our methodology allows for separation of calibration of idiosyncratic (marginal) laws of the underlying Markov process, from the calibration of the dependence structure of the process. The calibration really amounts to calibrating the infinitesimal generator of the underlying Markov process, and once this is done, the model can be used for consistent pricing and hedging of both the underlying products, such as CDO tranches, as well as options on such with future expiration dates; this feature obviously contributes to increased practical use of our methodology. In this sense, our Markov copula model is a genuine dynamic model, as a model of dependence between underlying stochastic processes. This not really the case with the model developed in [22], where the "dynamic copula" feature is in the sense of Patton's conditional copula [37, which is a stochastic process itself, and as such can't be calibrated to initial data.

- Last, but not least, the Markov copula approach of this paper is generic in the sense that, as demonstrated in [10, 11], it also applies to modeling of dynamics of credit ratings. This is not the case with the approach of [22].

Comparing now our methodology to the what is done in Brigo et al. [12, 13, 14], we see that the major differences can be summarized as follows:

- We are using a truly dynamic copula method, whereas in 12, 13, 14, a dynamic representation of essentially static copula - i.e. the Marshall-Olkin copula - is used.

- Our approach is a bottom-up approach, hence an approach applicable for hedging basket products using individual names, whereas the approach taken in [12, 13] is a top-down approach, and, as such, is not applicable for hedging basket products using individual names;

- This also applies to the so-called GPCL extension of the model of [14] in which individual names are represented of the model so that, in principle, hedging basket products using individual names could be considered in this setup. This is not practical however because fault of a suitable decoupling property between the dependence structure and the individual names in the model, the calibration of the model can only be addressed through a global joint optimization procedures involving all the model parameters at the same time, which is untractable numerically.

- Again, our approach is generic in the sense that it also applies to modeling of dynamics of credit ratings. This is not the case with the approach of [12, 13, 14].

The paper is organized as follows. In Section 2 we formulate a bottom-up Markovian copula model, in which individual default processes for various credit names are coupled together by means of simultaneous defaults. We then prove that conditionally on the full information in this model, the dependence structure of surviving names is equivalent to a Marshall-Olkin copula. In Section 3 we use this equivalence with the Marshall-Olkin framework in order to derive a common shocks model interpretation of our Markovian setting. This enables us to derive fast deterministic computational tractable algorithms for pricing and Greeking schemes in our heterogeneous model. In Section 4 we present numerical results of calibration against market data from CDO tranches as well as individual CDS spreads. We also discuss hedging sensitivities computed in the models thus calibrated. Technical 
proofs are deferred to Appendix A,

In the rest of the paper we consider a risk neutral pricing model $(\Omega, \mathcal{F}, \mathbb{P})$, for a filtration $\mathcal{F}=\left(\mathcal{F}_{t}\right)_{t \in[0, T]}$ which will be specified below and where $T \geq 0$ is a fixed time horizon. We denote $\mathbb{N}_{n}=\{1, \ldots, n\}$ and let $\mathcal{N}_{n}$ denote the set of all subsets of $\mathbb{N}_{n}$ where $n$ represents the number of obligors in the underlying credit portfolio. Further, we set $\max \emptyset=-\infty$.

\section{Model of Default Times}

In this section we construct a bottom-up Markovian model consisting of a multivariate factor process $\mathbf{X}$ and a vector $\mathbf{H}$ representing the default indicator processes in a pool of $n$ different credit names. More specifically, $\mathbf{H}_{t}$ is a vector in $\{0,1\}^{n}$ where the $i$-th entry of $\mathbf{H}_{t}$ is the indicator function for the event of a default of obligor $i$ up to time $t$. The purpose of the factor process $\mathbf{X}$ is to more realistically model diffusive randomness of credit spreads.

In our model defaults are the consequence of some "triggering-events" associated with groups of obligors. We then define the following pre-specified set of groups

$$
\mathcal{Y}=\left\{\{1\}, \ldots,\{n\}, I_{1}, \ldots, I_{m}\right\}
$$

where $I_{1}, \ldots, I_{m}$ are $m$ subsets of $\mathbb{N}_{n}$ (elements of the set $\mathcal{N}_{n}$ of all parts of $\mathbb{N}_{n}$ ), and each group $I_{l}$ contains at least two obligors or more. The triggering events are divided in two categories: the ones associated with singletons $\{1\}, \ldots,\{n\}$ can only trigger the default of name $1, \ldots, n$ individually, while the others associated with multi-name groups $I_{1}, \ldots, I_{m}$ may simultaneously trigger the default of all names in these groups. Note that several triggering events may affect the same particular name, so that only the one occurring first effectively triggers the default of that name. As a result, when a triggering-event associated with a specific group occurs at time $t$, it only triggers the default of names that are still alive in that group at time $t$. In the following, the elements $Y$ of $\mathcal{Y}$ will be used to designate triggering events and we let $\mathcal{I}=\left(I_{l}\right)_{1 \leq l \leq m}$ denote the pre-specified set of multi-name groups of obligors.

Let $\nu=|\mathcal{Y}|=n+m$ denote the cardinality of $\mathcal{Y}$. Given a multivariate Brownian motion $\mathbf{W}=\left(W^{Y}\right)_{Y \in \mathcal{Y}}$ with independent components, we assume that the factor process $\mathbf{X}=\left(X^{Y}\right)_{Y \in \mathcal{Y}}$ is a strong solution to

$$
d X_{t}^{Y}=b_{Y}\left(t, X_{t}^{Y}\right) d t+\sigma_{Y}\left(t, X_{t}^{Y}\right) d W_{t}^{Y}
$$

for suitable drift and diffusion functions $b_{Y}=b_{Y}(t, x)$ and $\sigma_{Y}=\sigma_{Y}(t, x)$. By application of Theorem 32 page 100 of Protter [38, this makes $\mathbf{X}$ an $\mathcal{F}^{\mathbf{W}}$-Markov process admitting the following generator acting on functions $v=v(t, \mathbf{x})$ with $\mathbf{x}=\left(x_{Y}\right)_{Y \in \mathcal{Y}}$

$$
A_{t} v(t, \mathbf{x})=\sum_{Y \in \mathcal{Y}}\left(b_{Y}\left(t, x_{Y}\right) \partial_{x_{Y}} v(t, \mathbf{x})+\frac{1}{2} \sigma_{Y}^{2}\left(t, x_{Y}\right) \partial_{x_{Y}^{2}}^{2} v(t, \mathbf{x})\right)
$$

Let $\mathcal{F}:=\mathcal{F}^{(\mathbf{W}, \mathbf{H})}$ be the filtration generated by the Brownian motion $\mathbf{W}$ and the point process $\mathbf{H}$. Given the "intensity functions" 1 of triggering-events, say $\lambda_{Y}=\lambda_{Y}\left(t, x_{Y}\right)$ for every triggering-event $Y \in \mathcal{Y}$, we would like to construct a model in which the $\mathcal{F}$-predictable intensity of a jump of $\mathbf{H}=\left(H^{i}\right)_{1 \leq i \leq n}$ from $\mathbf{H}_{t-}=\mathbf{k}$ to $\mathbf{H}_{t}=\mathbf{l}$, with $\mathbf{l} \neq \mathbf{k}$ in $\{0,1\}^{n}$, is

\footnotetext{
${ }^{1}$ These functions will indeed be interpreted as shock intensity functions in subsection 2.3 .
} 
given by

$$
\lambda\left(t, \mathbf{X}_{t}, \mathbf{k}, \mathbf{l}\right):=\sum_{\left\{Y \in \mathcal{Y} ; \mathbf{k}^{Y}=\mathbf{l}\right\}} \lambda_{Y}\left(t, X_{t}^{Y}\right),
$$

where, for any $Z \in \mathcal{N}_{n}$, the expression $\mathbf{k}^{Z}$ denotes the vector obtained from $\mathbf{k}=\left(k_{1}, \ldots, k_{n}\right)$ by replacing the components $k_{i}, i \in Z$, by numbers one (whenever $k_{i}$ is not equal to one already). The intensity of a jump of $\mathbf{H}$ from $\mathbf{k}$ to $\mathbf{l}$ at time $t$ is thus equal to the sum of the intensities of the triggering-events $Y \in \mathcal{Y}$ such that, if the joint default of the survivors in group $Y$ occurred at time $t$, then the state of $\mathbf{H}$ would move from $\mathbf{k}$ to $\mathbf{l}$.

Example 2.1 Figure 1] shows one possible defaults path in our model with $n=5$ and $\mathcal{Y}=\{\{1\},\{2\},\{3\},\{4\},\{5\},\{4,5\},\{2,3,4\},\{1,2\}\}$. The inner oval shows which commonshock triggering-event happened and caused the observed default scenarios at successive default times. At the first instant, default of name 2 is observed as the consequence of triggering-event $\{2\}$. At the second instant, names 4 and 5 have defaulted simultaneously as a consequence of triggering-event $\{4,5\}$. At the fourth instant, the triggering-event $\{2,3,4\}$ triggers the default of name 3 alone as name 2 and 4 have already defaulted. At the fifth instant, default of name 1 alone is observed as the consequence of triggering-event $\{1,2\}$. Note that the information produced by the arrival of the triggering-events cannot be deduced from the mere observation of the sequence of states followed by $\mathbf{H}_{t}$.

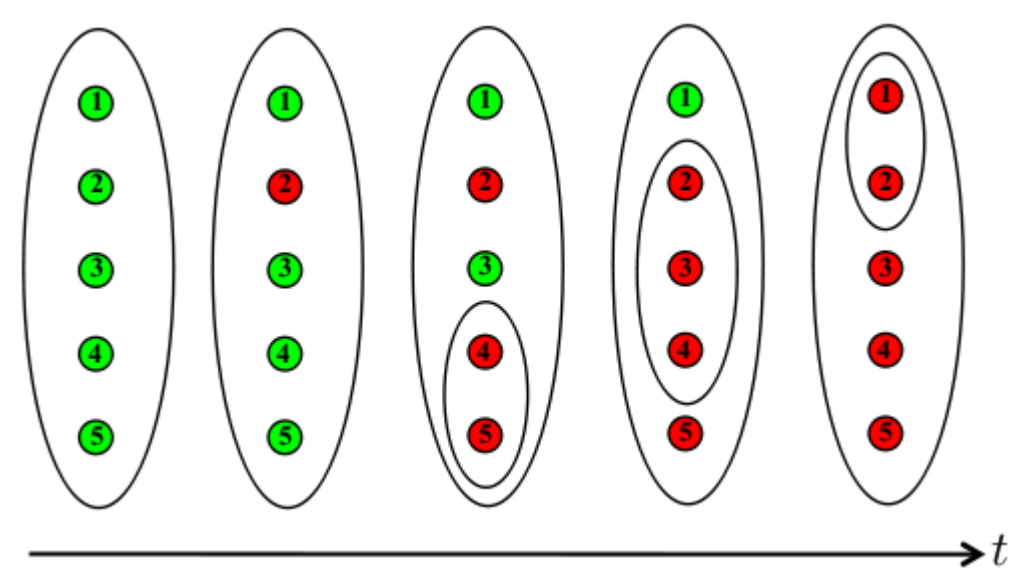

Figure 1: One possible defaults path in a model with $n=5$ and $\mathcal{Y}=$ $\{\{1\},\{2\},\{3\},\{4\},\{5\},\{4,5\},\{2,3,4\},\{1,2\}\}$.

To achieve (3) we follow the classical methodology: we construct $\mathbf{H}$ by an $\mathbf{X}$-related change of probability measure, starting from a continuous-time Markov chain with intensity one. This construction is detailed in Appendix A.1.

\subsection{Itô Formula}

In this subsection we state the Itô formula for functions of the Markov process $(\mathbf{X}, \mathbf{H})$.

For any set $Z \in \mathcal{N}_{n}$, let the set-event indicator process $H^{Z}$ denote the indicator process of a joint default of the names in $Z$ and only in $Z$. For $\mathbf{k}=\left(k_{1}, \ldots, k_{n}\right) \in\{0,1\}^{n}$, we introduce $\operatorname{supp}(\mathbf{k})=\left\{i \in \mathbb{N}_{n} ; k_{i}=1\right\}$ and $\operatorname{supp}^{c}(\mathbf{k})=\left\{i \in \mathbb{N}_{n} ; k_{i}=0\right\}$. Hence, $\operatorname{supp}(\mathbf{k})$ 
denotes the obligors who have defaulted in state $\mathbf{k}$ and similarly $\operatorname{supp}^{c}(\mathbf{k})$ are the survived names in the portfolio-state $\mathbf{k}$.

The following lemma provides the structure of the so called compensated set-event martingales $M^{Z}$, which we will use later as fundamental martingales to represent the pure jump martingale components of the various price processes involved.

Lemma 2.2 For every set $Z \in \mathcal{N}_{n}$ the intensity of $H^{Z}$ is given by $\ell_{Z}\left(t, \mathbf{X}_{t}, \mathbf{H}_{t}\right)$, so

$$
d M_{t}^{Z}=d H_{t}^{Z}-\ell_{Z}\left(t, \mathbf{X}_{t}, \mathbf{H}_{t}\right) d t
$$

is a martingale, and the set-event intensity function $\ell_{Z}(t, \mathbf{x}, \mathbf{k})$ is defined as

$$
\ell_{Z}(t, \mathbf{x}, \mathbf{k})=\sum_{Y \in \mathcal{Y} ; Y \cap \operatorname{supp}^{c}(\mathbf{k})=Z} \lambda_{Y}\left(t, x_{Y}\right)
$$

Proof. See Appendix A.1.1.

So $\ell_{Z}\left(t, \mathbf{X}_{t}, \mathbf{H}_{t-}\right)=\sum_{Y \in \mathcal{Y} ; Y_{t}=Z} \lambda_{Y}\left(t, X_{t}^{Y}\right)$, where for every $Y$ in $\mathcal{Y}=\left\{\{1\}, \ldots,\{n\}, I_{1}, \ldots, I_{m}\right\}$ we define

$$
Y_{t}=Y \cap \operatorname{supp}^{c}\left(\mathbf{H}_{t-}\right),
$$

the set-valued process representing the survived obligors in $Y$ right before time $t$. Let also $\mathcal{Z}_{t}=\left\{Z \in \mathbb{N}_{n} ; Z=Y_{t}\right.$ for at least one $\left.Y \in \mathcal{Y}\right\} \backslash \emptyset$ denote the set of all non-empty sets of survivors of sets $Y$ in $\mathcal{Y}$ right before time $t$.

We now derive a version of the Itô formula, which is relevant for our model. It will be used below for establishing the Markov properties of our set-up, as well as for deriving price dynamics. Let $\sigma(t, \mathbf{x})$ denote the diagonal matrix with diagonal $\left(\sigma_{Y}\left(t, x_{Y}\right)\right)_{Y \in \mathcal{Y}}$. Given a function $u=u(t, \mathbf{x}, \mathbf{k})$ with $\mathbf{x}=\left(x_{Y}\right)_{Y \in \mathcal{Y}}$ and $\mathbf{k}=\left(k_{i}\right)_{1 \leq i \leq n}$ in $\{0,1\}^{n}$, we denote

$$
\nabla u(t, \mathbf{x}, \mathbf{k})=\left(\partial_{x_{1}} u(t, \mathbf{x}, \mathbf{k}), \ldots, \partial_{x_{\nu}} u(t, \mathbf{x}, \mathbf{k})\right) .
$$

Let also $\delta u^{Z}$ represent the sensitivity of $u$ to the event $Z \in \mathcal{N}_{n}$, so

$$
\delta u^{Z}(t, \mathbf{x}, \mathbf{k})=u\left(t, \mathbf{x}, \mathbf{k}^{Z}\right)-u(t, \mathbf{x}, \mathbf{k}) .
$$

Proposition 2.3 Given a regular enough function $u=u(t, \mathbf{x}, \mathbf{k})$, one has

$$
\begin{gathered}
d u\left(t, \mathbf{X}_{t}, \mathbf{H}_{t}\right)=\left(\partial_{t}+\mathcal{A}_{t}\right) u\left(t, \mathbf{X}_{t}, \mathbf{H}_{t}\right) d t+\nabla u\left(t, \mathbf{X}_{t}, \mathbf{H}_{t}\right) \sigma\left(t, \mathbf{X}_{t}\right) d \mathbf{W}_{t} \\
+\sum_{Z \in \mathcal{Z}_{t}} \delta u^{Z}\left(t, \mathbf{X}_{t}, \mathbf{H}_{t-}\right) d M_{t}^{Z}
\end{gathered}
$$

where

$$
\begin{aligned}
\mathcal{A}_{t} u(t, \mathbf{x}, \mathbf{k})= & \sum_{Y \in \mathcal{Y}}\left(b_{Y}\left(t, x_{Y}\right) \partial_{x_{Y}} u(t, \mathbf{x}, \mathbf{k})+\frac{1}{2} \sigma_{Y}^{2}\left(t, x_{Y}\right) \partial_{x_{Y}^{2}}^{2} u(t, \mathbf{x}, \mathbf{k})\right) \\
& +\sum_{Y \in \mathcal{Y}} \lambda_{Y}\left(t, x_{Y}\right) \delta u^{Y}(t, \mathbf{x}, \mathbf{k}) .
\end{aligned}
$$


Proof. See Appendix A.1.2.

In the Itô formula (6) , the jump term may involve any of the $2^{n}$ set-events martingales $M^{Z}$ for $Z \in \mathcal{N}_{n}$. This suggests that the martingale dimension 2 of the model is $\nu+2^{n}$, where $\nu=n+m$ corresponds to the dimension of the Brownian motion $\mathbf{W}$ driving the factor process $\mathbf{X}$ and $2^{n}$ corresponds to the jump component $\mathbf{H}$. Yet by a reduction which is due to specific structure of the intensities in our set-up, the jump term of $\mathcal{A}_{t}$ in (77) is a sum over the set of triggering-events $\mathcal{Y}$, which has cardinality $\nu$.

Note that our model excludes direct contagion effects in which intensities of surviving names would be affected by past defaults, as opposed to the bottom-up contagion models treated by e.g. [16, 27, 28, 31]. To provide some understanding in this regard, we give a simple illustrative example.

Example 2.4 Take $\mathbb{N}_{n}=\{1,2,3\}$, so that the state space of $\mathbf{H}$ contains 8 elements:

$$
\{(0,0,0),(1,0,0),(0,1,0),(0,0,1),(1,1,0),(1,0,1),(0,1,1),(1,1,1)\} .
$$

Now, let $\mathcal{Y}$ be given as $\mathcal{Y}=\{\{1\},\{2\},\{3\},\{1,2\},\{1,2,3\}\}$. This is an example of the nested structure of $\mathcal{I}$ with $I_{1}=\{1,2\} \subset I_{2}=\{1,2,3\}$. Suppose for simplicity that $\lambda_{Y}$ does not depend either on $t$ or on $\mathbf{x}$ (dependence in $t, \mathbf{x}$ will be dealt with in Subsection 2.2). Then, the generator $\mathcal{A}$ of the chain $\mathbf{H}$ is given in matrix-form by

$$
\mathcal{A} \equiv\left[\begin{array}{cccccccc}
\cdot & \lambda_{\{1\}} & \lambda_{\{2\}} & \lambda_{\{3\}} & \lambda_{\{1,2\}} & 0 & 0 & \lambda_{\{1,2,3\}} \\
0 & \cdot & 0 & 0 & \lambda_{\{2\}}+\lambda_{\{1,2\}} & \lambda_{\{3\}} & 0 & \lambda_{\{1,2,3\}} \\
0 & 0 & \cdot & 0 & \lambda_{\{1\}}+\lambda_{\{1,2\}} & 0 & \lambda_{\{3\}} & \lambda_{\{1,2,3\}} \\
0 & 0 & 0 & . & 0 & \lambda_{\{1\}} & \lambda_{\{2\}} & \lambda_{\{1,2,3\}}+\lambda_{\{1,2\}} \\
0 & 0 & 0 & 0 & . & 0 & 0 & \lambda_{\{3\}}+\lambda_{\{1,2,3\}} \\
0 & 0 & 0 & 0 & 0 & \cdot & 0 & \lambda_{\{2\}}+\lambda_{\{1,2,3\}}+\lambda_{\{1,2\}} \\
0 & 0 & 0 & 0 & 0 & 0 & \cdot & \lambda_{\{1\}}+\lambda_{\{1,2,3\}}+\lambda_{\{1,2\}} \\
0 & 0 & 0 & 0 & 0 & 0 & 0 & 0
\end{array}\right]
$$

where '.' represents the sum of all other elements in the row multiplied with -1 . Now, consider group $\{1,2,3\}$. Suppose, that at some point of time obligor 2 is defaulted, but obligors 1 and 3 are still alive, so that process $\mathbf{H}$ is in state $(0,1,0)$. In this case the two survivors in the group $\{1,2,3\}$ may default simultaneously with intensity $\lambda_{\{1,2,3\}}$. Of course, here $\lambda_{\{1,2,3\}}$ cannot be interpreted as intensity of all three defaulting simultaneously, as obligor 2 has already defaulted. In fact, the only state of the model in which $\lambda_{\{1,2,3\}}$ can be interpreted as the intensity of all three defaulting, is state $(0,0,0)$. Note that obligor 1 defaults with intensity $\lambda_{\{1\}}+\lambda_{\{1,2,3\}}+\lambda_{\{1,2\}}$ regardless of the state of the pool, as long company 1 is alive. Similarly, obligor 2 will default with intensity $\lambda_{\{2\}}+\lambda_{\{1,2,3\}}+\lambda_{\{1,2\}}$ regardless of the state of the pool, as long company 1 is alive. Also, obligors 1 and 2 will default together with intensity $\lambda_{\{1,2,3\}}+\lambda_{\{1,2\}}$ regardless of the state of the pool, as long as company 1 and 2 still are alive.

\footnotetext{
${ }^{2}$ Minimal number of fundamental martingales which can be used as integrators to represent all the martingales in the model, see Appendix A.1
} 


\subsection{Markov Copula Properties}

Below, for every obligor $i$, a real-valued marginal factor process $X^{i}$ will be defined as a suitable function of the above multivariate factor process $\mathbf{X}=\left(X^{Y}\right)_{Y \in \mathcal{Y}}$. We shall then state conditions on the default intensities which enables us to prove that the marginal pair $\left(X^{i}, H^{i}\right)$ is a Markov process. Markovianity of the model marginals $\left(X^{i}, H^{i}\right)$ is crucial at the stage of calibration of the model, so that these marginals can be calibrated independently.

Observe that in view of (3), the intensity of a jump of $H^{i}$ from $H_{t-}^{i}=0$ to 1 is given by, for $t \in[0, T]$,

$$
\sum_{\{Y \in \mathcal{Y} ; i \in Y\}} \lambda_{Y}\left(t, X_{t}^{Y}\right)
$$

where the sum in this expression is taken over all pre-specified groups that contain name $i$. We define the marginal factor $X^{i}$ as a linear functional $\varphi_{i}$ of the multivariate factor process $\mathbf{X}=\left(X^{Y}\right)_{Y \in \mathcal{Y}}$ so that $X_{t}^{i}:=\varphi_{i}\left(\mathbf{X}_{t}\right)$. In general the transition intensity (9) implies nonMarkovianity of the marginal $\left(X^{i}, H^{i}\right)$. Hence, in order to make the process $\left(X^{i}, H^{i}\right)$ to be Markov, one needs to impose a more specified parametrization of (9) as well as conditions on the mapping $\varphi_{i}$. To be more specific:

Assumption 2.5 For every obligor $i$, there exists a linear form $\varphi_{i}(\mathbf{x})$ and a real-valued function $\lambda_{i}(t, x)$ such that for every $(t, \mathbf{x})$ with $\mathbf{x}=\left(x_{Y}\right)_{Y \in \mathcal{Y}}$

$$
\sum_{\{Y \in \mathcal{Y} ; i \in Y\}} \lambda_{Y}\left(t, x_{Y}\right)=\lambda_{i}\left(t, \varphi_{i}(\mathbf{x})\right),
$$

where, in addition, $X_{t}^{i}:=\varphi_{i}\left(\mathbf{X}_{t}\right)$ is a Markov-process with respect to the filtration $\mathcal{F}=$ $\mathcal{F}^{(\mathbf{W}, \mathbf{H})}$, with the following generator acting on functions $v_{i}=v_{i}(t, x)$ with $x \in \mathbb{R}$

$$
A_{t}^{i} v_{i}(t, x)=b_{i}(t, x) \partial_{x} v_{i}(t, x)+\frac{1}{2} \sigma_{i}^{2}(t, x) \partial_{x^{2}}^{2} v_{i}(t, x)
$$

for suitable drift and diffusion coefficients $b_{i}(t, x)$ and $\sigma_{i}(t, x)$.

Note that under such a specification of the intensities, dependence between defaults in the model does not only stem from the possibility of common jumps as in Example 2.4 but it can also come from the factor process $\mathbf{X}$ as in Example 2.7 below.

In the above assumption we require that $X_{t}^{i}=\varphi_{i}\left(\mathbf{X}_{t}\right)$ is a Markov process. This assumption is a non-trivial in general, as a process which is a measurable function of a Markov process does not have to be a Markov process itself. We refer to Pitman and Rogers [39] for some discussion of this issue. In our model set-up one, one can show that under appropriate regularity conditions, if for every $(t, \mathbf{x}, x)$ with $\mathbf{x}=\left(x_{Y}\right)_{Y \in \mathcal{Y}}$ and $x=\varphi_{i}(\mathbf{x})$, one has

$$
\begin{aligned}
& \sum_{\{Y \in \mathcal{Y}\}} b_{Y}(t, \mathbf{x}) \partial_{x_{Y}} \varphi_{i}(\mathbf{x})=b_{i}(t, x) \\
& \sum_{\{Y \in \mathcal{Y}\}} \sigma_{Y}^{2}(t, \mathbf{x})\left(\partial_{x_{Y}} \varphi_{i}(\mathbf{x})\right)^{2}=\sigma_{i}^{2}(t, x)
\end{aligned}
$$

then $X_{t}^{i}=\varphi_{i}\left(\mathbf{X}_{t}\right)$ is an $\mathcal{F}$-Markov process with generator $A^{i}$ in (11). The proof follows from Lemma A.2 (up to the reservation which is made right after the lemma regarding 
technicalities about the domain of the generators) since for every regular test-function $v_{i}=v_{i}(t, x)$, one has with $u(t, \mathbf{x}):=v_{i}\left(t, \varphi^{i}(\mathbf{x})\right)$

$$
\begin{aligned}
& v_{i}\left(t, X_{t}^{i}\right)-\int_{0}^{t}\left(\partial_{s}+A_{s}^{i}\right) v_{i}\left(s, X_{s}^{i}\right) d s \\
& \quad=u\left(t, \mathbf{X}_{t}\right)-\int_{0}^{t}\left(\partial_{s}+A_{s}\right) u\left(s, \mathbf{X}_{s}\right) d s .
\end{aligned}
$$

In the two examples given below, the $\mathcal{F}$-Markov property of $X_{t}^{i}=\varphi_{i}\left(\mathbf{X}_{t}\right)$ also rigorously follows, in case of Example2.6 where $\varphi_{i}$ is a coordinate projection operator, from the Markov consistency results of [8], or, in case of Example 2.7, from the semimartingale representation of $X^{i}$ provided by the SDE (14). The $\mathcal{F}$-Markov property of $X^{i}$ in Example 2.7 thus follows from the fact that a strong solution to the Markovian SDE (14) driven by the $\mathcal{F}$-Brownian motion $W^{i}$, is an $\mathcal{F}$-Markov process, by application of Theorem 32 page 100 of Protter [38. Example 2.7 is important, as it goes beyond the case of Example 2.6 where the $\lambda_{I}$ are deterministic functions of time, and it provides a fully stochastic specification of the $\lambda_{Y}$ (including the $\lambda_{I}$ ).

Example 2.6 (Deterministic Group Intensities) For every group $I \in \mathcal{I}$, the intensity $\lambda_{I}(t, \mathbf{x})$ does not depend on $\mathbf{x}$.

Letting $\varphi_{i}(\mathbf{x})=x_{\{i\}}$, then (10) and (12) hold with

$$
\begin{aligned}
\lambda_{i}(t, x) & :=\lambda_{\{i\}}(t, x)+\sum_{\{I \in \mathcal{I} ; i \in I\}} \lambda_{I}(t) \\
b_{i}(t, x) & :=b_{\{i\}}(t, x) \\
\sigma_{i}(t, x) & :=\sigma_{\{i\}}(t, x) .
\end{aligned}
$$

So, $X^{i}=X^{\{i\}}$ is $\mathcal{F}$-Markov with drift and diffusion coefficients $b_{i}(t, x)$ and generator $\sigma_{i}(t, x)$ thus specified.

Example 2.7 (Extended CIR Intensities) For every $Y \in \mathcal{Y}$, the pre-specified group intensities are given by $\lambda_{Y}\left(t, X_{t}^{Y}\right)=X_{t}^{Y}$, where the factor $X^{Y}$ is an extended CIR process

$$
d X_{t}^{Y}=a\left(b_{Y}(t)-X_{t}^{Y}\right) d t+c \sqrt{X_{t}^{Y}} d W_{t}^{Y}
$$

for non-negative constants $a, c$ and non-negative functions $b_{Y}(t)$. The SDE-s for the factors $X^{Y}$ have thus the same coefficients except for the $b_{Y}(t)$.

$$
\begin{aligned}
& \text { Letting } \varphi_{i}(\mathbf{x})=\sum_{\{Y \in \mathcal{Y} ; i \in Y\}} x_{Y}=x_{\{i\}}+\sum_{\{I \in \mathcal{I} ; i \in I\}} x_{I} \text {, and denoting likewise } b_{i}(t)= \\
& \sum_{\{Y \in \mathcal{Y} ; i \in Y\}} b_{Y}(t)=b_{\{i\}}(t)+\sum_{\{I \in \mathcal{I} ; i \in I\}} b_{I}(t), \text { then (10) and (12) hold with } \\
& \lambda_{i}(t, x):=x \\
& b_{i}(t, x):=a\left(b_{i}(t)-x\right) \\
& \sigma_{i}(t, x):=c \sqrt{x} .
\end{aligned}
$$

So, $X^{i}=\sum_{\{Y \in \mathcal{Y} ; i \in Y\}} X^{Y}$ is an $\mathcal{F}$-Markov process with drift and diffusion coefficients $b_{i}(t, x)$ and generator $\sigma_{i}(t, x)$ thus specified. 
Note that $X^{i}$ satisfies the following extended CIR SDE with parameters $a, b_{i}(t)$ and $c$ as

$$
d X_{t}^{i}=a\left(b_{i}(t)-X_{t}^{i}\right) d t+c \sqrt{X_{t}^{i}} d W_{t}^{i}
$$

for the $\mathcal{F}$-Brownian motion $W^{i}$ such that

$$
\sqrt{X_{t}^{i}} d W_{t}^{i}=\sum_{i \in Y} \sqrt{X_{t}^{Y}} d W_{t}^{Y}, d W_{t}^{i}=\sum_{i \in Y} \frac{\sqrt{X_{t}^{Y}}}{\sqrt{\sum_{i \in Y} X_{t}^{Y}}} d W_{t}^{Y} .
$$

Remark 2.8 Both the time-deterministic group intensities specification of Example 2.6 and the affine intensities specification of Example 2.7 have already been fruitfully used in the context of various credit and counterparty credit risk applications (anticipating the theoretical aspects of the model which are dealt with in the present paper), see [7, 2, 6].

For every $Y \in \mathcal{Y}$ and every set of non-negative constants $t_{i}$, we define the quantities $\Lambda_{s, t}^{Y}, \Lambda_{t}^{Y}$ and $\theta_{t}^{Y}$ as

$$
\Lambda_{s, t}^{Y}=\int_{s}^{t} \lambda_{Y}\left(s, X_{s}^{Y}\right) d s, \Lambda_{t}^{Y}=\Lambda_{0, t}^{Y}=\int_{0}^{t} \lambda_{Y}\left(s, X_{s}^{Y}\right) d s \quad \text { and } \quad \theta_{t}^{Y}=\max _{i \in Y \cap \operatorname{supp}^{c}\left(\mathbf{H}_{t}\right)} t_{i}
$$

where $Y \cap \operatorname{supp}^{c}\left(\mathbf{H}_{t}\right)$ in $\theta_{t}^{Y}$ is the set of survivors in $Y$ at time $t$ (and we use in $\theta_{t}^{Y}$ our convention that $\max \emptyset=-\infty)$. Note that $\Lambda^{I}$ is a deterministic function of time for every group $I \in \mathcal{I}$. Let $\tau_{i}$ denote the default time for obligor $i$. Since $H^{i}$ is the default indicator of name $i$, we have

$$
\tau_{i}=\inf \left\{t>0 ; H_{t}^{i}=1\right\}, H_{t}^{i}=\mathbb{1}_{\left\{\tau_{i} \leq t\right\}} .
$$

The following Proposition gathers the Markov properties of the model.

Proposition 2.9 (i) $(\mathbf{X}, \mathbf{H})$ is an $\mathcal{F}$-Markov process with infinitesimal generator given by $\mathcal{A}$.

(ii) For every obligor $i,\left(X^{i}, H^{i}\right)$ is an $\mathcal{F}$-Markov proces 43 admitting the following generator acting on functions $u_{i}=u_{i}\left(t, x_{i}, k_{i}\right)$ with $\left(x_{i}, k_{i}\right) \in \mathbb{R} \times\{0,1\}$

$$
\begin{gathered}
\mathcal{A}_{t}^{i} u_{i}\left(t, x_{i}, k_{i}\right)=b_{i}\left(t, x_{i}\right) \partial_{x_{i}} u_{i}\left(t, x_{i}, k_{i}\right)+\frac{1}{2} \sigma_{i}^{2}\left(t, x_{i}\right) \partial_{x_{i}^{2}}^{2} u_{i}\left(t, x_{i}, k_{i}\right) \\
+\lambda_{i}\left(t, x_{i}\right)\left(u_{i}\left(t, x_{i}, 1\right)-u_{i}\left(t, x_{i}, k_{i}\right)\right) .
\end{gathered}
$$

Moreover, the $\mathcal{F}$-intensity proces@ $44^{4}$ of $H^{i}$ given by $\mathbb{1}_{\left\{\tau_{i}>t\right\}} \lambda_{i}\left(t, X_{t}^{i}\right)$. In other words, the process $M^{i}$ defined by

$$
M_{t}^{i}=\mathbb{1}_{\left\{\tau_{i} \leq t\right\}}-\int_{0}^{t} \mathbb{1}_{\left\{\tau_{i}>s\right\}} \lambda_{i}\left(s, X_{s}^{i}\right) d s,
$$

is an $\mathcal{F}$-martingale 5

(iii) For any fixed non-negative constants $t, t_{1}, \ldots, t_{n}$, one has

$$
\begin{gathered}
\mathbb{P}\left(\tau_{1}>t_{1}, \ldots, \tau_{n}>t_{n} \mid \mathcal{F}_{t}\right)=\mathbb{P}\left(\tau_{1}>t_{1}, \ldots, \tau_{n}>t_{n} \mid \mathbf{H}_{t}, \mathbf{X}_{t}\right) \\
=\mathbb{1}_{\left\{t_{i}<\tau_{i}, i \in \operatorname{supp}\left(\mathbf{H}_{t}\right)\right\}} \mathbb{E}\left\{\exp \left(-\sum_{Y \in \mathcal{Y}} \Lambda_{t, \theta_{t}^{Y}}^{Y}\right) \mid \mathbf{X}_{t}\right\} .
\end{gathered}
$$

\footnotetext{
${ }^{3}$ And hence an $\mathcal{F}^{\left(X^{i}, H^{i}\right)}$-Markov process.

${ }^{4}$ And hence, $\mathcal{F}^{\left(X^{i}, H^{i}\right)}$-intensity process.

${ }^{5}$ And hence, an $\mathcal{F}^{\left(X^{i}, H^{i}\right)}$-martingale.
} 
The conditional survival probability function of every obligor $i$ is given by, for every $t_{i} \geq t$,

$$
\begin{aligned}
\mathbb{P}\left(\tau_{i}>t_{i} \mid \mathcal{F}_{t}\right) & =\mathbb{P}\left(\tau_{i}>t_{i} \mid \mathbf{H}_{t}, \mathbf{X}_{t}\right) \\
& =\mathbb{1}_{\left\{\tau_{i}>t\right\}} \mathbb{E}\left\{\exp \left(-\sum_{Y \in \mathcal{Y}, i \in Y} \Lambda_{t, t_{i}}^{Y}\right) \mid \mathbf{X}_{t}\right\} \\
& =\mathbb{1}_{\left\{\tau_{i}>t\right\}} \mathbb{E}\left\{\exp \left(-\int_{t}^{t_{i}} \lambda_{i}\left(s, X_{s}^{i}\right) d s\right) \mid X_{t}^{i}\right\} \\
& =\mathbb{1}_{\left\{\tau_{i}>t\right\}} G_{t}^{i}\left(t_{i}\right),
\end{aligned}
$$

with

$$
G_{t}^{i}\left(t_{i}\right)=\mathbb{E}\left\{\exp \left(-\int_{t}^{t_{i}} \lambda_{i}\left(s, X_{s}^{i}\right) d s\right) \mid X_{t}^{i}\right\}
$$

In particular

$$
\mathbb{E}\left\{\exp \left(-\Lambda_{t}^{\{i\}}\right)\right\}=\exp \left\{-\left(\Gamma_{i}(t)-\sum_{i \in I} \Lambda_{t}^{I}\right)\right\},
$$

where $\Gamma_{i}(t)=-\ln G_{0}^{i}(t)=-\ln \left(\mathbb{P}\left(\tau_{i}>t\right)\right)$ is the hazard function of name $i$.

Proof. See Appendix A.2.1.

We shall illustrate part (iii) of the above proposition using the following example.

Example 2.10 In case of two obligors and $\mathcal{Y}=\{\{1\},\{2\},\{1,2\}\}$, one can easily check that (17) boils down to

$$
\begin{gathered}
\mathbb{P}\left(\tau_{1}>t_{1}, \tau_{2}>t_{2} \mid \mathcal{F}_{t}\right)=\mathbb{1}_{\left\{\tau_{1}>t\right\}} \mathbb{1}_{\left\{\tau_{2}>t\right\}} \mathbb{E}\left\{\exp \left(-\sum_{Y \in \mathcal{Y}} \int_{t}^{t_{1} \vee t_{2}} \lambda_{Y}\left(s, \mathbf{X}_{s}\right)\right) \mid \mathbf{X}_{t}\right\} \\
+\mathbb{1}_{\left\{t_{2}<\tau_{2} \leq t\right\}} \mathbb{1}_{\left\{\tau_{1}>t\right\}} \mathbb{E}\left\{\exp \left(-\int_{t}^{t_{1}} \lambda_{1}\left(s, X_{s}^{1}\right) d s\right) \mid X_{t}^{1}\right\} \\
+\mathbb{1}_{\left\{t_{1}<\tau_{1} \leq t\right\}} \mathbb{1}_{\left\{\tau_{2}>t\right\}} \mathbb{E}\left\{\exp \left(-\int_{t}^{t_{2}} \lambda_{2}\left(s, X_{s}^{2}\right) d s\right) \mid X_{t}^{2}\right\} \\
+\mathbb{1}_{\left\{t_{1}<\tau_{1} \leq t\right\}} \mathbb{1}_{\left\{t_{2}<\tau_{2} \leq t\right\}} .
\end{gathered}
$$

\subsection{Common Shocks Model Interpretation}

In this subsection we establish a connection between the dynamic Markovian model $(\mathbf{X}, \mathbf{H})$, and a common shock model with a Marshall-Olkin common factor structure of default times as in Lindskog and McNeil [35], Elouerkhaoui [22] or Brigo et al. [12, 13, 14].

In rough terms, conditionally on any given state $(\mathbf{x}, \mathbf{k})$ of $(\mathbf{X}, \mathbf{H})$ at time $t$, it is possible to define a common shock model of default times of the surviving names at time $t$, such that the conditional law of the default times in the common shock model is the same as the corresponding conditional distribution in the original Markov model. This connection between the Markovian model and the common shock framework is the main theoretical contribution of this paper. 
We thus introduce a family of common shocks copula models, parameterized by the current time $t$. For every $Y \in \mathcal{Y}$, we define

$$
\tau_{Y}(t)=\inf \left\{\theta>t ; \Lambda_{\theta}^{Y}>\Lambda_{t}^{Y}+E_{Y}\right\},
$$

where the random variables $E_{Y}$ are i.i.d. and exponentially distributed with parameter 1 . For every obligor $i$ we let

$$
\tau_{i}(t)=\min _{\{Y \in \mathcal{Y} ; i \in Y\}} \tau_{Y}(t),
$$

which defines the default time of obligor $i$ in the common shocks copula model starting at time $t$. We also introduce the common shock model indicator processes $H_{\theta}^{Y}(t)=\mathbb{1}_{\left\{\tau_{Y}(t) \leq \theta\right\}}$ and $H_{\theta}^{i}(t)=\mathbb{1}_{\left\{\tau_{i}(t) \leq \theta\right\}}$, for every triggering-event $Y$, obligor $i$ and time horizon $\theta \geq t$. Let $Z \in \mathcal{N}_{n}$ denote a set of obligors, meant in the probabilistic interpretation to represent the set $\operatorname{supp}^{c}\left(\mathbf{H}_{t}\right)$ of survived obligors in the Markov model at time $t$. We now prove that on $\left\{\operatorname{supp}^{c}\left(\mathbf{H}_{t}\right)=Z\right\}$, the conditional law of $\left(\tau_{i}\right)_{i \in \operatorname{Supp}^{c}\left(\mathbf{H}_{t}\right)}$ given $\mathcal{F}_{t}$ in the Markov model, is equal to the conditional law of $\left(\tau_{i}(t)\right)_{i \in Z}$ given $\mathbf{X}_{t}$ in the common shocks framework. Let also $N_{\theta}=\sum_{1 \leq i \leq n} H_{\theta}^{i}$ denote the cumulative number of defaulted obligors in the Markov model up to time $\theta$. Let $N_{\theta}(t, Z)=n-|Z|+\sum_{i \in Z} H_{\theta}^{i}(t)$, denote the cumulative number of defaulted obligors in the common shocks framework up to time $\theta$ where $|Z|$ is the cardinality of the set $Z$.

Proposition 2.11 Let $Z \in \mathcal{N}_{n}$ denote an arbitrary subset of obligors and let $t \geq 0$. Then, (i) for every $t_{1}, \ldots, t_{n} \geq t$, one has

$$
\mathbb{1}_{\left\{\operatorname{supp}^{c}\left(\mathbf{H}_{t}\right)=Z\right\}} \mathbb{P}\left(\tau_{i}>t_{i}, i \in \operatorname{supp}^{c}\left(\mathbf{H}_{t}\right) \mid \mathcal{F}_{t}\right)=\mathbb{1}_{\left\{\operatorname{supp}^{c}\left(\mathbf{H}_{t}\right)=Z\right\}} \mathbb{P}\left(\tau_{i}(t)>t_{i}, i \in Z \mid \mathbf{X}_{t}\right) .
$$

(ii) for every $\theta \geq t$, one has that for every $k=n-|Z|, \ldots, n$,

$$
\mathbb{1}_{\left\{\operatorname{supp}\left(\mathbf{H}_{t}\right)=Z\right\}} \mathbb{P}\left(N_{\theta}=k \mid \mathcal{F}_{t}\right)=\mathbb{1}_{\left\{\operatorname{suppc}\left(\mathbf{H}_{t}\right)=Z\right\}} \mathbb{P}\left(N_{\theta}(t, Z)=k \mid \mathbf{X}_{t}\right) .
$$

Proof. Part (ii) readily follows from part (i), that we now show. Let, for every obligor $i$, $\tilde{t}_{i}=\mathbb{1}_{i \in \operatorname{Supp}^{c}\left(\mathbf{H}_{t}\right)} t_{i}$. Note that one has, for $Y \in \mathcal{Y}$

$$
\max _{i \in Y \cap \operatorname{supp}^{c}\left(\mathbf{H}_{t}\right)} \tilde{t}_{i}=\max _{i \in Y \cap \operatorname{supp}^{c}\left(\mathbf{H}_{t}\right)} t_{i}=\theta_{t}^{Y}
$$

Thus, by application of identity (17) in Proposition 2.9 to the sequence of times $\left(\tilde{t}_{i}\right)_{1 \leq i \leq n}$, it comes,

$$
\begin{aligned}
& \mathbb{1}_{\left\{\operatorname{supp}^{c}\left(\mathbf{H}_{t}\right)=Z\right\}} \mathbb{P}\left(\tau_{i}>t_{i}, i \in \operatorname{supp}^{c}\left(\mathbf{H}_{t}\right) \mid \mathcal{F}_{t}\right) \\
& =\mathbb{1}_{\left\{\operatorname{supp}^{c}\left(\mathbf{H}_{t}\right)=Z\right\}} \mathbb{P}\left(\left(\tau_{i}>t_{i}, i \in Z\right),\left(\tau_{i}>0, i \in Z^{c}\right) \mid \mathcal{F}_{t}\right) \\
& =\mathbb{1}_{\left\{\operatorname{supp}^{c}\left(\mathbf{H}_{t}\right)=Z\right\}} \mathbb{E}\left\{\exp \left(-\sum_{Y \in \mathcal{Y}} \Lambda_{t, \theta_{t}^{Y}}^{Y}\right) \mid \mathbf{X}_{t}\right\}
\end{aligned}
$$

which on $\left\{\operatorname{supp}^{c}\left(\mathbf{H}_{t}\right)=Z\right\}$ coincides with the expression

$$
\mathbb{E}\left\{\exp \left(-\sum_{Y \in \mathcal{Y}} \Lambda_{t, \max _{i \in Y \cap Z} t_{i}}^{Y}\right) \mid \mathbf{X}_{t}\right\}
$$


derived for $\mathbb{P}\left(\tau_{i}(t)>t_{i}, i \in Z \mid \mathbf{X}_{t}\right)$ in the common shocks model of Elouerkhaoui [22].

For instance, in the situation of Example 2.4, the shock interpretation at time $t=0$ is clear: there are five different shocks, corresponding to the elements of $\mathcal{Y}$. In particular, obligors 1 and 2 can default simultaneously if either the shock corresponding to $\{1,2\}$ arrives, or the shock corresponding to $\{1,2,3\}$ arrives.

This interpretation will be used in the next section for deriving fast exact convolution recursion procedures for pricing portfolio loss derivatives.

The common shocks interpretation can also be used for simulation purposes. In view of Proposition 2.11(i) and given $\mathcal{F}_{t}$, the simulation of the random times $\left(\tau_{i}\right)_{i \in \operatorname{Supp}^{c}\left(\mathbf{H}_{t}\right)}$, or equivalently on $\left\{\operatorname{supp}^{c}\left(\mathbf{H}_{t}\right)=Z\right\},\left(\tau_{i}(t)\right)_{i \in Z}$, is fast. One essentially needs to simulate IID exponential random variables $E_{Y}$.

\section{Pricing, Calibration and Hedging Issues}

This section treats the pricing, calibration and hedging issues in the Markov copula model of Section 2. First, in Subsection 3.1 we derive the price dynamics for CDS contracts and for CDO tranches in this model. In Subsection 3.2 we use dynamics of Subsection 3.1 to derive min-variance hedging strategies in the Markov copula model. In the case of CDO$\mathrm{s}$ these formulas lead to a very large PDE-system which in practice is difficult to solve. So, in Subsection 3.3 we instead exploit the relationship between our Markov model and the common shock model, which enables us to derive fast, deterministic, computationally tractable algorithms for derivation of the prices and sensitivities.

For notational convenience, we assume zero interest rates. The extension of all theoretical results to time dependent, deterministic interest rates is straightforward but more cumbersome notationally, especially regarding hedging. Time-dependent deterministic interest rates will be used in the numerical part.

\subsection{Pricing Equations}

In this subsection we derive price dynamics formulas for CDS contracts and CDO tranches in the Markov model; all prices are considered from perspective of the protection buyers. These dynamics will be useful when deriving the min-variance hedging strategies in Subsection 3.2 .

In a zero interest-rates environment, the (ex-dividend) price process of an asset is simply given by the risk neutral conditional expectation of future cash flows associated with the asset; the cumulative value process is the sum of the price process and of the cumulative cash-flows process. The cumulative value process is a martingale, as opposed to the price process. When it comes to hedging, the cumulative value process is the main quantity of interest (see for instance Frey and Backhaus [24]).

For a fixed maturity $T$, we let $S_{i}$ denote the $T$-year CDS spread for obligor $i$, with recovery rate $R_{i}$. Similarly, we let $S$ denote the $T$-year model CDO tranche spread for the tranche $[a, b]$, with payoff process

$$
L_{t}^{a, b}=L_{a, b}\left(\mathbf{H}_{t}\right)=\left(L_{t}-a\right)^{+}-\left(L_{t}-b\right)^{+},
$$

where $L_{t}=\frac{1}{n} \sum_{i=1}^{n}\left(1-R_{i}\right) H_{t}^{i}$ is the credit loss process for the underlying portfolio. The premium legs in these products are payed at $t_{1}<t_{2}<\ldots<t_{p}=T$ where $t_{j}-t_{j-1}=\mathrm{h}$ and $\mathrm{h}$ is typically a quarter. Below, the notation is the same as in the Itô formula (6). 
Proposition 3.1 (i) The price $P^{i}$ and the cumulative value $\widehat{P}^{i}$ at time $t \in[0, T]$ of the single-name $C D S$ on obligor $i$ with contractual spread $S_{i}$ are given by

$$
\begin{aligned}
& P_{t}^{i}=\mathbb{1}_{\left\{\tau_{i}>t\right\}} v_{i}\left(t, X_{t}^{i}\right) \\
& d \widehat{P}_{t}^{i}=\mathbb{1}_{\left\{\tau_{i}>t\right\}} \partial_{x_{i}} v_{i}\left(t, X_{t}^{i}\right) \sigma_{i}\left(t, X_{t}^{i}\right) d W_{t}^{i}+\sum_{Z \in \mathcal{Z}_{t}} \mathbf{1}_{i \in Z}\left(1-R_{i}-v_{i}\left(t, X_{t}^{i}\right)\right) d M_{t}^{Z}
\end{aligned}
$$

for a pre-default pricing function $v_{i}\left(t, x_{i}\right)$ such that

$$
\mathbb{1}_{\left\{\tau_{i}>t\right\}} v_{i}\left(t, X_{t}^{i}\right)=\mathbb{E}\left[-S_{i} \mathrm{~h} \sum_{t<t_{j} \leq T} \mathbb{1}_{\left\{\tau_{i}>t_{j}\right\}}+\left(1-R_{i}\right) \mathbb{1}_{\left\{t<\tau_{i} \leq T\right\}} \mid \mathcal{F}_{t}\right] .
$$

(ii) The price process $\Pi$ and cumulative value $\widehat{\Pi}$ at time $t \in[0, T]$ of a CDO tranche $[a, b]$ with contractual spread $S$ are given by

$$
\begin{aligned}
& \Pi_{t}=u\left(t, \mathbf{X}_{t}, \mathbf{H}_{t}\right) \\
& \begin{aligned}
d \widehat{\Pi}_{t}=\nabla u\left(t, \mathbf{X}_{t}, \mathbf{H}_{t}\right) \sigma\left(t, \mathbf{X}_{t}\right) d \mathbf{W}_{t} \\
\quad+\sum_{Z \in \mathcal{Z}_{t}}\left(L_{a, b}\left(\mathbf{H}_{t-}^{Z}\right)-L_{a, b}\left(\mathbf{H}_{t-}\right)+\delta u^{Z}\left(t, \mathbf{X}_{t}, \mathbf{H}_{t-}\right)\right) d M_{t}^{Z}
\end{aligned}
\end{aligned}
$$

for a pricing function $u(t, \mathbf{x}, \mathbf{k})$ such that

$$
u\left(t, \mathbf{X}_{t}, \mathbf{H}_{t}\right)=\mathbb{E}\left[-S \mathrm{~h} \sum_{t<t_{j} \leq T}\left(b-a-L_{t_{j}}^{a, b}\right)+L_{T}^{a, b}-L_{t}^{a, b} \mid \mathcal{F}_{t}\right] .
$$

Proof. See Appendix A.2.2.

Note that in view of the marginal Markov property of the model, the martingale representation (24) of $\widehat{P}^{i}$ can be reduced to a "univariate" martingale representation based on the compensated martingale $M^{i}$ of $H^{i}$ in (16). However, as will be clear from Subsection 3.2. it is more useful to state martingale representations of $\widehat{\Pi}$ and $\widehat{P}^{i}$ relatively to a common set of fundamental martingales in order to handle the hedging issue.

The pricing functions $v_{i}$ and $u$ can be characterized as the unique solutions to the related Kolmogorov equations (68) and (170) in Appendix A.2.2. If the pricing functions are known, the prices at a given time are recovered by plugging the corresponding state of the model into the right-hand-side of the first lines of (24) or (25). The pricing equation (70) for a CDO tranche leads to a large system of PDEs which in practice is impossible to handle numerically as soon as $n$ is larger than a few units. As a remedy for this we will in Subsection 3.3 instead use the translation to a Marshall-Olkin framework which allows us to derive practical recursive pricing schemes for CDO tranche price processes.

\subsection{Min-Variance Hedging}

In this subsection we use the price dynamics from Subsection 3.1 to derive min-variance hedging strategies in the Markov copula model. By min-variance hedging strategies we mean strategies that minimize the variance of the hedging error. Note that one could also try to minimize the variance relatively to the historical probability measure, however in this paper we minimize the risk-neutral variance for simplicity: see Schweizer [40] for a survey about various quadratic hedging approaches. The hedging strategies are theoretically sound 
due to our bottom-up Markovian framework and they will be shown in Subsection 3.3 to be computationally tractable thanks to the Marshall-Olkin copula interpretation of Subsection 2.3 .

Consider a CDO tranche $[a, b]$ with pricing function $u$ specified in Proposition 3.1. Our aim is to find explicit min-variance hedging formulas when hedging this CDO tranche by using the savings account and $d$ single-name CDSs with pricing functions $v_{i}$ given by Proposition 3.1. First we introduce the CDS cumulative value vector-function

$$
\mathbf{v}(t, \mathbf{x}, \mathbf{k})=\left(\mathbb{1}_{k_{1}=0} v_{1}\left(t, x_{1}\right)+\mathbb{1}_{k_{1}=1}\left(1-R_{1}\right), \ldots, \mathbb{1}_{k_{d}=0} v_{d}\left(t, x_{d}\right)+\mathbb{1}_{k_{d}=1}\left(1-R_{d}\right)\right)^{\top} .
$$

Let $\nabla \mathbf{v}$ denote the Jacobian matrix of $\mathbf{v}$ with respect to $\mathbf{x}$ in the sense of the $d \times \nu$-matrix such that $\nabla \mathbf{v}(t, \mathbf{x}, \mathbf{k})_{i}^{j}=\mathbb{1}_{k_{j}=0} \partial_{x_{j}} v_{i}\left(t, x_{i}\right)$, for every $1 \leq i \leq d$ and $1 \leq j \leq \nu$. Let $\Delta \mathbf{v}^{Z}$ represent the vector-function of the sensitivities of $v$ with respect to the event $Z \in \mathcal{N}_{n}$, so $\Delta \mathbf{v}^{Z}(t, \mathbf{x}, \mathbf{k})=\left(\mathbb{1}_{1 \in Z, k_{1}=0}\left(\left(1-R_{1}\right)-v_{1}\left(t, x_{1}\right)\right), \ldots, \mathbb{1}_{d \in Z, k_{d}=0}\left(\left(1-R_{d}\right)-v_{d}\left(t, x_{d}\right)\right)\right)^{\top}$.

By using the vector notation $\widehat{\mathbf{P}}=\left(\widehat{P}^{i}\right)_{1 \leq i \leq d}$, one has in view of Proposition 3.1(i)

$$
d \widehat{\mathbf{P}}_{t}=\nabla \mathbf{v}\left(t, \mathbf{X}_{t}, \mathbf{H}_{t}\right) \sigma\left(t, \mathbf{X}_{t}\right) d \mathbf{W}_{t}+\sum_{Z \in \mathcal{Z}_{t}} \Delta \mathbf{v}^{Z}\left(t, \mathbf{X}_{t}, \mathbf{H}_{t-}\right) d M_{t}^{Z}
$$

Let

$$
\Delta u^{Z}(t, \mathbf{x}, \mathbf{k})=\delta^{Z} u(t, \mathbf{x}, \mathbf{k})+L_{a, b}\left(\mathbf{k}^{Z}\right)-L_{a, b}(\mathbf{k}) .
$$

represent the function of sensitivity of the CDO tranche $[a, b]$ cumulative value process with respect to the event $Z \in \mathcal{N}_{n}$. Let $\zeta$ be an $d$-dimensional row-vector process, representing the number of units held in the first $d$ CDSs which are used in a self-financing 6 hedging strategy for the CDO tranche $[a, b]$. Given (25) and (26)), the tracking error $\left(e_{t}\right)$ of the hedged portfolio satisfies $e_{0}=0$ and, for $t \in[0, T]$

$$
\begin{aligned}
d e_{t}= & d \widehat{\Pi}_{t}-\zeta_{t} d \widehat{\mathbf{P}}_{t} \\
= & \left(\nabla u\left(t, \mathbf{X}_{t}, \mathbf{H}_{t}\right)-\zeta_{t} \nabla \mathbf{v}\left(t, \mathbf{X}_{t}, \mathbf{H}_{t}\right)\right) \sigma\left(t, \mathbf{X}_{t}\right) d \mathbf{W}_{t} \\
& \quad+\sum_{Z \in \mathcal{Z}_{t}}\left(\Delta u^{Z}\left(t, \mathbf{X}_{t}, \mathbf{H}_{t-}\right)-\zeta_{t} \Delta \mathbf{v}^{Z}\left(t, \mathbf{X}_{t}, \mathbf{H}_{t-}\right)\right) d M_{t}^{Z}
\end{aligned}
$$

Since the martingale dimension of the model is $\nu+2^{n}$, replication is typically out-of-reach 7 in the Markov model. However, in view of (27), we still can find min-variance hedging formulas.

Proposition 3.2 The min-variance hedging strategy $\zeta$ is

$$
\zeta_{t}=\frac{d\langle\widehat{\Pi}, \widehat{\mathbf{P}}\rangle_{t}}{d t}\left(\frac{d\langle\widehat{\mathbf{P}}\rangle_{t}}{d t}\right)^{-1}=\zeta\left(t, \mathbf{X}_{t}, \mathbf{H}_{t-}\right)
$$

where $\zeta=(u, \mathbf{v})(\mathbf{v}, \mathbf{v})^{-1}$, with

$$
\begin{aligned}
& (u, \mathbf{v})=(\nabla u) \sigma^{2}(\nabla \mathbf{v})^{\top}+\sum_{Y \in \mathcal{Y}} \lambda_{Y} \Delta u^{Y}\left(\Delta \mathbf{v}^{Y}\right)^{\top} \\
& (\mathbf{v}, \mathbf{v})=(\nabla \mathbf{v}) \sigma^{2}(\nabla \mathbf{v})^{\top}+\sum_{Y \in \mathcal{Y}} \lambda_{Y} \Delta \mathbf{v}^{Y}\left(\Delta \mathbf{v}^{Y}\right)^{\top} .
\end{aligned}
$$

\footnotetext{
${ }^{6}$ Using also the savings account (constant asset).

${ }^{7}$ See the comments following Proposition 2.3
} 
Proof. The first identity in (28) is a classical risk neutral min-variance hedging 8 formula, derived for instance in Section 3.5 of Crépey [18]. Moreover, one has by computation of the oblique brackets based on the second lines in (24) and (25):

$$
\begin{aligned}
& \frac{d\langle\widehat{\Pi}, \widehat{\mathbf{P}}\rangle_{t}}{d t}=\left((\nabla u) \sigma^{2}(\nabla \mathbf{v})^{\top}+\sum_{Z \in \mathcal{Z}_{t}} \lambda_{Z} \Delta u^{Z}\left(\Delta \mathbf{v}^{Z}\right)^{\top}\right)\left(t, \mathbf{X}_{t}, \mathbf{H}_{t-}\right)=(u, \mathbf{v})\left(t, \mathbf{X}_{t}, \mathbf{H}_{t-}\right) \\
& \frac{d\langle\widehat{\mathbf{P}}\rangle_{t}}{d t}=\left((\nabla \mathbf{v}) \sigma^{2}(\nabla \mathbf{v})^{\top}+\sum_{Z \in \mathcal{Z}_{t}} \lambda_{Z} \Delta \mathbf{v}^{Z}\left(\Delta \mathbf{v}^{Z}\right)^{\top}\right)\left(t, \mathbf{X}_{t}, \mathbf{H}_{t-}\right)=(\mathbf{v}, \mathbf{v})\left(t, \mathbf{X}_{t}, \mathbf{H}_{t-}\right)
\end{aligned}
$$

where the second identities in both lines of (30) use simplifications similar to those used in the proof of the Itô formula (6) in Appendix A.1.2.

In (29), the $u$-related terms can be computed by using the conditional convolutionrecursion procedures presented in Subsection 3.3, the $v_{i}$-related terms can be computed very quickly (actually semi-explicitly in either of the specifications of examples 2.6 and 2.7). We will illustrate in Subsection 4.5 the tractability of this approach for computing min-variance hedging deltas.

We refer the reader to Elouerkhaoui [22] for analogous results in the context of the common shock model presented in Subsection 2.3. A nice feature of our set-up however is that due to the specific structure of the intensities, the sums in (29) are over the set $\mathcal{Y}$ of triggering-events $\mathcal{Y}$ which is of cardinality $\nu=n+m$ rather than over the set $\mathcal{N}_{n}$ of all set-events $Z$, which would be of cardinality $2^{n}$.

We also refer the reader to Frey and Backhaus 24] for other related min-variance hedging formulas.

\subsection{Convolution Recursion Pricing Schemes}

In this subsection we use the common shock model interpretation to derive fast convolution recursion algorithms for computing the portfolio loss distribution. In the case where the recovery rate is the same for all names, i.e., $R_{i}=R, i=1, \ldots, n$, the aggregate loss $L_{t}$ at time $t$ is equal to $(1-R) N_{t}$, where we recall $N_{t}$ is the total number of defaults that have occurred in the Markov model up to time $t$. It is well known, see, e.g., [15, 24, 26, 31, and Proposition 3.1(ii), that the price process for a CDO tranche $[a, b]$ is determined by the probabilities $\mathbb{P}\left[N_{\theta}=k \mid \mathcal{F}_{t}\right]$ for $k=\left|\mathbf{H}_{t}\right|, \ldots, n$ and $\theta \geq t \geq 0$. Thanks to the common shock model interpretation of Subsection 2.3, one has from Proposition 2.11(ii) that

$$
\mathbb{P}\left[N_{\theta}=k \mid \mathcal{F}_{t}\right]=\mathbb{P}\left[N_{\theta}(t, Z)=k \mid \mathbf{X}_{t}\right]
$$

on the event $\left\{\operatorname{supp}^{c}\left(\mathbf{H}_{t}\right)=Z\right\}$, so we will focus on computation of the latter probabilities, which are derived in formula (32) below. Furthermore, recall that $\operatorname{supp}^{c}\left(\mathbf{H}_{t}\right)$ denotes the obligors who have survived in state $\mathbf{H}_{t}$ at time $t$.

We henceforth assume a nested structure of the sets $I_{j}$ given by

$$
I_{1} \subset \ldots \subset I_{m}
$$

This structure implies that if all obligors in group $I_{k}$ have defaulted, then all obligors in group $I_{1}, \ldots, I_{k-1}$ have also defaulted. As we shall detail in Remark [3.4, the nested

\footnotetext{
${ }^{8}$ See Schweizer 40
} 
structure (31) yields a particularly tractable expression for the portfolio loss distribution. This nested structure also makes sense financially with regards to the hierarchical structure of risks which is reflected in standard CDO tranches.

Remark 3.3 A dynamic group structure would be preferable from a financial point of view. In the same vein one could deplore the absence of direct contagion effects in this model (it only has joint defaults). However it should be stressed that we are building a pricing model, not an econometric model; the applications we have in mind are hedging CDO tranches by individual names (see Subsection 4.5), as well as valuation and hedging of counterparty risk on credit portfolios (see [6]). In these regards, efficient pricing (at any future point in time, not only at time 0 [6]) and Greeking procedures, as well as efficient joint calibration to CDS and CDO data (see Subsections 4.1 and 4.3), are the main issues, and these are already quite difficult to achieve simultaneously in a single model.

Denoting conventionally $I_{0}=\emptyset$ and $H_{\theta}^{I_{0}}(t)=1$, then in view of (31), the events

$$
\Omega_{\theta}^{j}(t):=\left\{H_{\theta}^{I_{j}}(t)=1, H_{\theta}^{I_{j+1}}(t)=0, \ldots, H_{\theta}^{I_{m}}(t)=0\right\}, 0 \leq j \leq m
$$

form a partition of $\Omega$. Hence, we have

$$
\mathbb{P}\left(N_{\theta}(t, Z)=k \mid \mathbf{X}_{t}\right)=\sum_{0 \leq j \leq m} \mathbb{P}\left(N_{\theta}(t, Z)=k \mid \Omega_{\theta}^{j}(t), \mathbf{X}_{t}\right) \mathbb{P}\left(\Omega_{\theta}^{j}(t) \mid \mathbf{X}_{t}\right)
$$

where, by construction of the $H_{\theta}^{I}(t)$ and independence of the $\lambda_{I}\left(t, X_{t}^{I}\right)$ we have

$$
\mathbb{P}\left(\Omega_{\theta}^{j}(t) \mid \mathbf{X}_{t}\right)=\left(1-\mathbb{E}\left(e^{-\Lambda_{t, \theta}^{I_{j}}} \mid X_{t}^{I_{j}}\right)\right) \prod_{j+1 \leq l \leq m} \mathbb{E}\left(e^{-\Lambda_{t, \theta}^{I_{l}}} \mid X_{t}^{I_{l}}\right)
$$

which in our Markov setup can be computed very quickly (actually, semi-explicitly in either of the specifications of examples 2.6 and 2.7). We now turn to the computation of the term

$$
\mathbb{P}\left(N_{\theta}(t, Z)=k \mid \Omega_{\theta}^{j}(t), \mathbf{X}_{t}\right)
$$

appearing in (32). Recall first that $N_{\theta}(t, Z)=n-|Z|+\sum_{i \in Z} H_{\theta}^{i}(t)$ with $|Z|$ denoting the cardinality of $Z$. We know that for every group $j=1, \ldots, m$, given $\Omega_{\theta}^{j}(t)$, the marginal default indicators $H_{\theta}^{i}(t)$ for $i \in Z$ are such that:

$$
H_{\theta}^{i}(t)= \begin{cases}1, & i \in I_{j} \\ H_{\theta}^{\{i\}}(t), & \text { else. }\end{cases}
$$

Hence, the $H_{\theta}^{i}(t)$ are conditionally independent given $\Omega_{\theta}^{j}(t)$. Finally, conditionally on $\left(\Omega_{\theta}^{j}(t), \mathbf{X}_{t}\right)$ the random vector $\mathbf{H}_{\theta}(t)=\left(H_{\theta}^{i}(t)\right)_{i \in \mathbb{N}_{n}}$ is a vector of independent Bernoulli random variables with parameter $p=\left(p_{\theta}^{i, j}(t)\right)_{i \in \mathbb{N}_{n}}$, where

$$
p_{\theta}^{i, j}(t)= \begin{cases}1, & i \in I_{j}, \\ 1-\mathbb{E}\left\{\exp \left(-\Lambda_{t, \theta}^{\{i\}}\right) \mid X_{t}^{\{i\}}\right\}, & \text { else }\end{cases}
$$

The conditional probability (34) can therefore be computed by a standard convolution recursive procedure (see, for instance, Andersen and Sidenius [1]). 
Remark 3.4 The linear number of terms in the sum of (32) is due to the nested structure of the groups $I_{j}$ in (32). Note that a convolution recursion procedure is possible for an arbitrary structuring of the groups $I_{j}$. However, a general structuring of the $m$ groups $I_{j}$ would imply $2^{m}$ terms instead of $m$ in the sum of (32), which in practice would only work for very few groups $m$. The nested structure (32) of the $I_{j}$, or equivalently, the tranched structure of the $I_{j} \backslash I_{j-1}$, is also quite natural from the point of view of application to CDO tranches.

\subsection{Random Recoveries}

In this subsection we outline how to modify the Markov copula to include stochastic recoveries. The implementation details are here omitted and we refer the readers to [5] for a more comprehensive description of the methodology. Let $\mathbf{L}=\left(L^{i}\right)_{1 \leq i \leq n}$ represent the $[0,1]^{n}$ valued vector process of the loss given defaults in the pool of names. The process $\mathbf{L}$ is a multivariate process where $\mathbf{L}_{0} \in \mathbf{0}$, and where each component $L_{t}^{i}$ represents the fractional loss that name $i$ may have suffered due to default until time $t$. Assuming unit notional for each name, the cumulative loss process for the entire portfolio is given by $L_{t}=\frac{1}{n} \sum_{i=1}^{n} L_{t}^{i}$. We assume that all obligors $i$ have i.i.d. distribution for the recovery. The default times are defined as before, but at every time of jump of $\mathbf{H}$, an independent recovery draw is made for every newly defaulted name $i$, determining the recovery $R_{i}$ of name $i$. In particular, the recovery rates resulting from a joint default are thus drawn independently for the affected names.

Independent recoveries do not break the Markovian nor the Markovian copula structure. However by introducing stochastic recoveries we can no longer use the exact convolution recursion procedures of Subsection 3.3 for pricing CDO tranches. Instead we will here use an approximate procedure based on the exponential approximations of the so called hockey stick function, as presented in Iscoe et al. [32], 33] and originally developed by [34]. We now briefly outline how to use this method for computing the price of a CDO tranche in our Markov model conditionally on $\mathcal{F}_{t}$.

Recall that the tranche-loss function $L_{t}^{a, b}$ for the tranche $[a, b]$ as a function of the portfolio credit loss $L_{t}$ is given by $L_{t}^{a, b}=\left(L_{t}-a\right)^{+}-\left(L_{t}-b\right)^{+}$. This function can in turn be rewritten as (see e.g [32])

$$
L_{t}^{a, b}=b\left(1-h\left(\frac{L_{t}}{b}\right)\right)-a\left(1-h\left(\frac{L_{t}}{a}\right)\right)
$$

where $h(x)$ is the so-called hockey stick function given by

$$
h(x)= \begin{cases}1-x & \text { if } 0 \leq x \leq 1 \\ 0 & \text { if } 1<x\end{cases}
$$

Next, 34 shows that for any fixed $\epsilon>0$, the function $h(x)$ can be approximated by a function $h_{\exp }^{(q)}(x)$ on $[0, d]$ for any $d>0$ so that $\left|h(x)-h_{\exp }^{(q)}(x)\right| \leq \epsilon$ for all $x \in[0, d]$ where $q=q(\epsilon)$ is positive integer and $h_{\exp }^{(q)}(x)$ is given by

$$
h_{\exp }^{(q)}(x)=\sum_{\ell=1}^{q} \omega_{\ell} \exp \left(\gamma_{\ell} \frac{x}{d}\right) .
$$

Here $\left(\omega_{\ell}\right)_{\ell=1}^{q}$ and $\left(\gamma_{\ell}\right)_{\ell=1}^{q}$ are complex numbers obtained as roots of polynomials whose coefficients can be computed numerically in a straightforward way. Figure 2 visualizes the 
approximation $h_{\exp }^{(q)}(x)$ of $h(x)$ for $d=2$ on $x \in[0,10]$ with $q=5$ and $q=50$ as well as the approximation error $\left|h(x)-h_{\exp }^{(q)}(x)\right|$ for the same $q$. As can be seen in Figure 2, the approximation is fairly good already for small values values of $q$ and also works well outside the interval $[0, d]=[0,2]$, that is on the interval $(2,10]$.
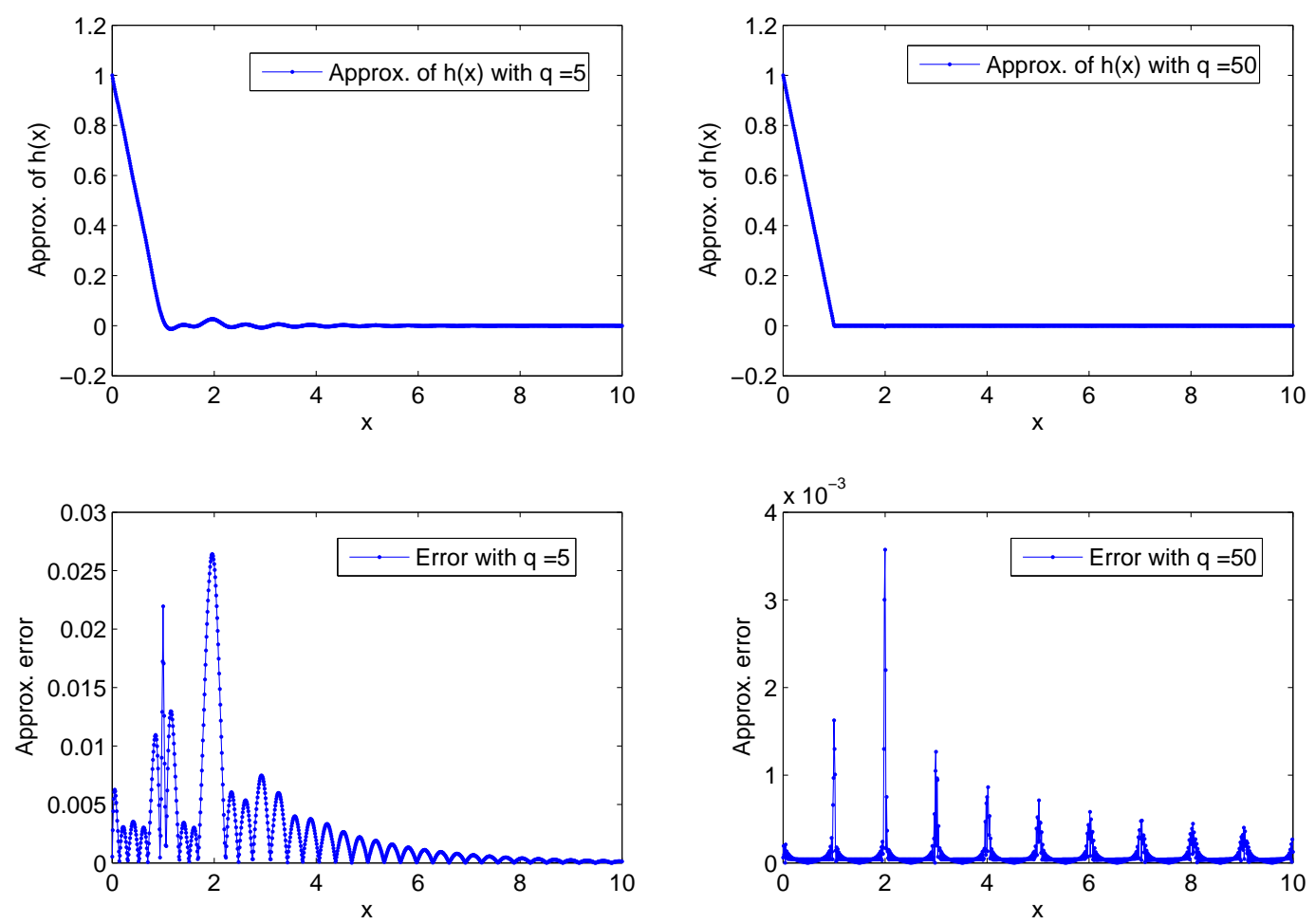

Figure 2: The function $h_{\exp }^{(q)}(x)$ as approximation of $h(x)$ for $x \in[0,10]$ with $q=5$ and $q=50$ (top) and the corresponding approximation errors (bottom).

In [32], 33] the authors chooses $d=2$ for (39) in all their numerical implementations, see also [5]. In the rest of this paper we will use $d=2$ in (39).

In view of (37)-(39) for any two time points $\theta>t$, the conditional pricing of a CDO tranche given the information $\mathcal{F}_{t}$ at any time $t$, boils down to computation of conditional expectations of the form

$$
\mathbb{E}\left(e^{\gamma_{\ell} \frac{L_{\theta}}{2 c}} \mid \mathcal{F}_{t}\right)
$$

for $\ell=1,2, \ldots, q$ and different attachment points $c$ and time horizons $\theta>t$. Note that the case $t=0$ is used in the calibration, while the case $t>0$ with $\theta>t$ is needed for pricing the credit valuation adjustment (CVA) on a CDO tranche in a counterparty risky environment, a topical issue since the 2007-09 credit crisis (see [19]). Since the algorithm for computing $\mathbb{E}\left(e^{\gamma_{\ell} \frac{L_{\theta}}{2 c}} \mid \mathcal{F}_{t}\right)$ is the same for each $\ell=1,2, \ldots, q$ and any attachment point $c$, we will below for notational convenience simply write $\mathbb{E}\left(e^{\gamma L_{\theta}} \mid \mathcal{F}_{t}\right)$ instead of $\mathbb{E}\left(e^{\gamma_{\ell} \frac{L_{\theta}}{2 c}} \mid \mathcal{F}_{t}\right)$.

Now, by the common shock model interpretation as in Subsection 3.3 (and using the 
same notation that was introduced there), one has much like in Proposition 2.11(ii) that for every $Z \in \mathcal{N}_{n}$

$$
\mathbb{1}_{\left\{\operatorname{supp}^{c}\left(\mathbf{L}_{t}\right)=Z\right\}} \mathbb{E}\left(e^{\gamma L_{\theta}} \mid \mathcal{F}_{t}\right)=\mathbb{1}_{\left\{\operatorname{supp}^{c}\left(\mathbf{L}_{t}\right)=Z\right\}} \mathbb{E}\left(e^{\gamma L_{\theta}(t, Z)} \mid \mathbf{X}_{t}\right)
$$

where $L_{\theta}(t, Z):=\sum_{i \notin Z} L_{t}^{i}+\sum_{i \in Z}\left(1-R_{i}\right) H_{\theta}^{i}(t)$ and $H_{\theta}^{i}(t)$ is defined as in (35). Furthermore, $R_{i}$ is a random recovery with values in $[0,1]$. One then has as in (32) that

$$
\mathbb{E}\left(e^{\gamma L_{\theta}(t, Z)} \mid \mathbf{X}_{t}\right)=\sum_{0 \leq j \leq m} \mathbb{E}\left(e^{\gamma L_{\theta}(t, Z)} \mid \Omega_{\theta}^{j}(t), \mathbf{X}_{t}\right) \mathbb{P}\left(\Omega_{\theta}^{j}(t) \mid \mathbf{X}_{t}\right)
$$

where $\mathbb{P}\left(\Omega_{\theta}^{j}(t) \mid \mathbf{X}_{t}\right)$ is given by (33). Moreover by conditional independence one has on $\mathbb{1}_{\left\{\operatorname{supp}^{c}\left(\mathbf{L}_{t}\right)=Z\right\}}$ that

$$
\begin{aligned}
& \mathbb{E}\left(e^{\gamma L_{\theta}(t, Z)} \mid \Omega_{\theta}^{j}(t), \mathbf{X}_{t}\right) \\
& =e^{\gamma \sum_{i \notin Z} L_{t}^{i}} \mathbb{E}\left(e^{\gamma \sum_{i \in Z}\left(1-R_{i}\right) H_{\theta}^{i}(t)} \mid \Omega_{\theta}^{j}(t), \mathbf{X}_{t}\right) \\
& =e^{\gamma \sum_{i \notin Z} L_{t}^{i}} \prod_{i \in Z} \mathbb{E}\left(e^{\gamma\left(1-R_{i}\right) H_{\theta}^{i}(t)} \mid \Omega_{\theta}^{j}(t), \mathbf{X}_{t}\right) .
\end{aligned}
$$

Now observe that for every $i$

$$
\mathbb{E}\left(e^{\gamma\left(1-R_{i}\right) H_{\theta}^{i}(t)} \mid \Omega_{\theta}^{j}(t), \mathbf{X}_{t}\right)= \begin{cases}\mathbb{E}\left(e^{\gamma\left(1-R_{i}\right)}\right), & i \in I_{j}, \\ \mathbb{E}\left(e^{\gamma\left(1-R_{i}\right) H_{\theta}^{\{i\}}(t)} \mid X_{t}^{\{i\}}\right), & \text { else }\end{cases}
$$

in which by independence of $R_{i}$ and $H_{\theta}^{i}(t)$ implies that

$$
\mathbb{E}\left(e^{\gamma\left(1-R_{i}\right) H_{\theta}^{\{i\}}(t)} \mid X_{t}^{\{i\}}\right)=1-p_{\theta}^{i, j}(t)\left(1-\mathbb{E} e^{\gamma\left(1-R_{i}\right)}\right)
$$

where $p_{\theta}^{i, j}(t)$ was defined in (36).

In Subsection 4.2 we will give an explicitly example of the recovery rate $R_{i}$ which will be used with the above hockey-stick method when calibrating the Markov copula against market data on CDO tranches. As will be seen in Subsection 4.3, using stochastic recoveries will for some data sets render much better calibration results compared with the case of using constant recoveries.

\section{Numerical Results}

In this section we briefly discuss the calibration of the model and some few numerical results connected to the loss-distributions and the min-variance hedging. Subsection 4.1 outlines the calibration methodology with piecewise constant default intensities and constant recoveries while Subsection 4.2 describes the calibration procedure with stochastic recoveries and piecewise constant default intensities. Then Subsection 4.3 presents the numerical calibration of the Markov copula model against market data both with constant and stochastic recoveries. We also study the implied loss-distributions in our fitted model for the case with constant recoveries. Furthermore, in Subsection 4.4, we consider that individual and joint defaults are driven by stochastic default intensities and we describe the calibration methodology and results for a particular model specification. Finally, Subsection 4.5 discusses min-variance hedging sensitivities in the calibrated models using constant recoveries. A more extensive numerical study of the model can be found in the paper [5]. 


\subsection{Calibration Methodology with Piecewise Constant Default Intensities and Constant Recoveries}

In this subsection we discuss one of the calibration methodologies that will be used when fitting the Markov copula model against CDO tranches on the iTraxx Europe and CDX.NA.IG series in Subsection 4.3. This first calibration methodology will use piecewise constant default intensities and constant recoveries in the convolution pricing algorithm of Subsection 3.3 .

The first step is to calibrate the single-name CDS for every obligor. Given the $T$-year market CDS spread $S_{i}^{*}$ for obligor we want to find the individual default parameters for obligor $i$ so that $P_{0}^{i}\left(S_{i}^{*}\right)=0$, or in view of Proposition 3.1(i),

$$
S_{i}^{*}=\frac{\left(1-R_{i}\right) \mathbb{P}\left(\tau_{i}<T\right)}{\mathrm{h} \sum_{0<t_{j} \leq T} \mathbb{P}\left(\tau_{i}>t_{j}\right)}
$$

where we used the facts that interest rate is zero and that the recovery $R_{i}$ is constant. Hence, the first step is to extract the implied hazard function $\Gamma_{i}^{*}(t)$ in (20) from the CDS curve of every obligor $i$ by using a standard bootstrapping procedure based on (45).

Given the marginal hazard functions, the law of the total number of defaults $N$ at a fixed time $t$ is a function of the joint default intensity functions $\lambda_{I}(t)$, as described by the recursive algorithm of Subsection 3.3. The second step is therefore to calibrate the common-shock intensities $\left\{\lambda_{I}(t)\right\}$ so that the model CDO tranche spreads coincide with the corresponding market spreads. This is done by using the recursive algorithm of Subsection 3.3, for $\lambda_{I}(t)$-s parameterized as non-negative and piecewise constant functions of time. Moreover, in view of (20), for every obligor $i$ and at each time $t$ we impose the constraint

$$
\sum_{I \in \mathcal{I} ; i \in I} \lambda_{I}(t) \leq \lambda_{i}^{*}(t)
$$

where $\lambda_{i}^{*}:=\frac{d \Gamma_{i}^{*}}{d t}$ denotes the hazard rate (or hazard intensity) of name $i$. For constant joint default intensities $\lambda_{I}(t)=\lambda_{I}$ the constraints (46) reduce to

$$
\sum_{I \ni i} \lambda_{I} \leq \underline{\lambda}_{i}:=\inf _{t \in[0, T]} \lambda_{i}^{*}(t) \text { for every obligor } i .
$$

Given the nested structure of the groups $I_{j}$-s specified in (31), this is equivalent to

$$
\sum_{j=l}^{m} \lambda_{I_{j}} \leq \underline{\lambda}_{I_{l}}:=\min _{i \in I_{l} \backslash I_{l-1}} \underline{\lambda}_{i} \text { for every group } l .
$$

Furthermore, for piecewise constant common shock intensities on a time grid $\left(T_{k}\right)$, the condition (47) extends to the following constraint

$$
\sum_{j=l}^{m} \lambda_{I_{j}}^{k} \leq \underline{\lambda}_{I_{l}}^{k}:=\min _{i \in I_{l} \backslash I_{l-1}} \underline{\lambda}_{i}^{k} \quad \text { for every } l, k \quad \text { where } \underline{\lambda}_{i}^{k}:=\inf _{t \in\left[T_{k-1}, T_{k}\right]} \lambda_{i}^{*}(t) .
$$

We remark that insisting on calibrating all CDS names in the portfolio, including the safest ones, implies via (47) or (48) a very constrained region for the common shock parameters. This region can be expanded by relaxing the system of constraints for the joint default intensities, by excluding the safest CDSs from the calibration. 
In this paper we will use a time grid consisting of two maturities $T_{1}$ and $T_{2}$. Hence, the single-name CDSs constituting the entities in the credit portfolio are bootstrapped from their market spreads for $T=T_{1}$ and $T=T_{2}$. This is done by using piecewise constant individual default intensity $\lambda_{i}$-s on the time intervals $\left[0, T_{1}\right]$ and $\left[T_{1}, T_{2}\right]$.

Before we leave this subsection, we give some few more details on the calibration of the common shock intensities for the $m$ groups in the second calibration step. From now on we assume that the joint default intensities $\left\{\lambda_{I_{j}}(t)\right\}_{j=1}^{m}$ are piecewise constant functions of time, so that $\lambda_{I_{j}}(t)=\lambda_{I_{j}}^{(1)}$ for $t \in\left[0, T_{1}\right]$ and $\lambda_{I_{j}}(t)=\lambda_{I_{j}}^{(2)}$ for $t \in\left[T_{1}, T_{2}\right]$ and for every group $j$. Next, the joint default intensities $\boldsymbol{\lambda}=\left(\lambda_{I_{j}}^{(k)}\right)_{j, k}=\left\{\lambda_{I_{j}}^{(k)}: j=1, \ldots, m\right.$ and $\left.k=1,2\right\}$ are then calibrated so that the five-year model spread $S_{a_{l}, b_{l}}(\boldsymbol{\lambda})=: S_{l}(\boldsymbol{\lambda})$ will coincide with the corresponding market spread $S_{l}^{*}$ for each tranche $l$. To be more specific, the parameters $\boldsymbol{\lambda}=\left(\lambda_{I_{j}}^{(k)}\right)_{j, k}$ are obtained according to

$$
\boldsymbol{\lambda}=\underset{\widehat{\boldsymbol{\lambda}}}{\operatorname{argmin}} \sum_{l}\left(\frac{S_{l}(\widehat{\boldsymbol{\lambda}})-S_{l}^{*}}{S_{l}^{*}}\right)^{2}
$$

under the constraints that all elements in $\boldsymbol{\lambda}$ are nonnegative and that $\boldsymbol{\lambda}$ satisfies the inequalities (48) for every group $I_{l}$ and in each time interval $\left[T_{k-1}, T_{k}\right]$ where $T_{0}=0$. In $S_{l}(\widehat{\boldsymbol{\lambda}})$ we have emphasized that the model spread for tranche $l$ is a function of $\boldsymbol{\lambda}=\left(\lambda_{I_{j}}^{(k)}\right)_{j, k}$ but we suppressed the dependence in other parameters like interest rate, payment frequency or $\lambda_{i}$, $i=1, \ldots, n$. In the calibration we used an interest rate of $3 \%$, the payments in the premium leg were quarterly and the integral in the default leg was discretized on a quarterly mesh. For each data-set we use a constant recovery of $40 \%$. We use MatLab in our numerical calculations and the objective function (49) is minimized by using the built in optimization routine fmincon together with the constraints given by equations on the form (48).

In Subsection 4.3 we use the above setting for our two data-set and perform a calibration with constant recovery of $40 \%$.

\subsection{Calibration Methodology with Piecewise Constant Default Intensities and Stochastic Recoveries}

In this subsection we discuss the second calibration methodology used when fitting the Markov copula model against CDO tranches on the iTraxx Europe and CDX.NA.IG series in Subsection 4.3. This method relies on piecewise constant default intensities and stochastic recoveries. Recall that compared with constant recoveries, using stochastic recoveries requires a more sophisticated method in order to compute the tranche loss distribution, as was explained in Subsection 3.4. The methodology and constraints connected to the piecewise constant default intensities are the same as in Subsection 4.1. Therefore we will in this subsection only discuss the distribution for the individual stochastic recoveries $R_{i}$ as well as accompanying constraints used in the calibration. This distribution will determine the quantity $\mathbb{E}\left(e^{\gamma\left(1-R_{i}\right)}\right)$ in (44) which is needed to compute the tranche loss distribution.

We assume that the individual recoveries $\left\{R_{i}\right\}$ are i.i.d and have a binomial mixture distribution on the following form

$$
R_{i} \sim \frac{1}{K} \operatorname{Bin}\left(K, R^{*}\left(p_{0}+(1-\Theta) p_{1}\right)\right) \text { where } \Theta \in\{0,1\} \text { and } \mathbb{P}[\Theta=1]=q
$$

where $R^{*}, q p_{0}$ and $p_{1}$ are positive constants and $K$ is an integer (in this paper we let 
$K=10$ ). As a result, the recovery rate distribution function is given by

$$
\mathbb{P}\left[R_{i}=\frac{k}{K}\right]=\sum_{\xi=0}^{1} \mu(\xi)\left(\begin{array}{l}
K \\
k
\end{array}\right) p(\xi)^{k}(1-p(\xi))^{K-k} \quad \text { where } \quad p(\xi)=R^{*}\left(p_{0}+(1-\xi) p_{1}\right)
$$

for $\xi \in\{0,1\}$ and $\mu(1)=q, \mu(0)=1-q$. Let the constant $R^{*}$ in (50) represent the average recovery for each obligor in the portfolio, which we assume to be the same for all obligors. We next impose the constraint $\mathbb{E}\left[R_{i}\right]=R^{*}$ which is necessary in order to have a calibration of the single-name CDSs that is separate from the calibration of the common-shock parameters. The condition $\mathbb{E}\left[R_{i}\right]=R^{*}$ leads to the following constraint on the parameters $q$ (see in [5] for a detailed derivation)

$$
q<\min \left(1, \frac{1}{p_{0}}, \frac{1-R^{*}}{1-R^{*} p_{0}}\right)
$$

Furthermore, the constraint $\mathbb{E}\left[R_{i}\right]=R^{*}$ also implies that $p_{1}=\frac{1-p_{0}}{1-q}$ so $p_{1}$ can be seen as a function of $q$ and $p_{0}$. The constraints (52) will be used in our calibration of the CDO tranches simultaneously with the other constraints for the common shock intensities. In our calibrations the parameters $p_{0}$ and $R^{*}$ will be treated as exogenously given parameters where we set $R^{*}=40 \%$ while $p_{0}$ can be any positive scalar satisfying $p_{0}<\frac{1}{R^{*}}$. The scalar $p_{0}$ will give us some freedom to fine-tune our calibrations. A more detailed description of the constraints for $p_{0}, q$ and $p_{1}$ are given in [5].

In this subsection we thus combine the stochastic recoveries in (50) with piecewise constant default intensities as described in Subsection 4.1 so the parameters to be calibrated will be on the form $\boldsymbol{\theta}=(\boldsymbol{\lambda}, q)$ where $\boldsymbol{\lambda}$ are the same as in Subsection 4.1. Consequently, using the same notation as in Subsection 4.1 the parameters $\boldsymbol{\theta}=(\boldsymbol{\lambda}, q)$ are obtained according to

$$
\boldsymbol{\theta}=\underset{\widehat{\boldsymbol{\theta}}}{\operatorname{argmin}} \sum_{l}\left(\frac{S_{l}(\widehat{\boldsymbol{\theta}})-S_{l}^{*}}{S_{l}^{*}}\right)^{2}
$$

where $\boldsymbol{\lambda}$ must satisfies the same constraints as in Subsection 4.1 while $q$ must obey (52). The rest of the notation in (53) are defined as in Subsection 4.1. In Subsection 4.3 we use the above setting with stochastic recoveries when calibrating this model against two different CDO data-sets.

Finally, note that if the i.i.d recoveries $R_{i}$ would follow other distributions than (50) we simply modify $\mathbb{E} e^{\gamma\left(1-R_{i}\right)}$ in (44) in Subsection 3.4 but the rest of the computations are the same. Of course, changing (50) will also imply that the constraints in (52) will no longer be relevant.

\subsection{Calibration Results with Piecewise Constant Default Intensities}

In this subsection we calibrate our model against CDO tranches on the iTraxx Europe and CDX.NA.IG series with maturity of five years. We use the calibration methodology described in Subsection 4.1 and Subsection 4.2 . 


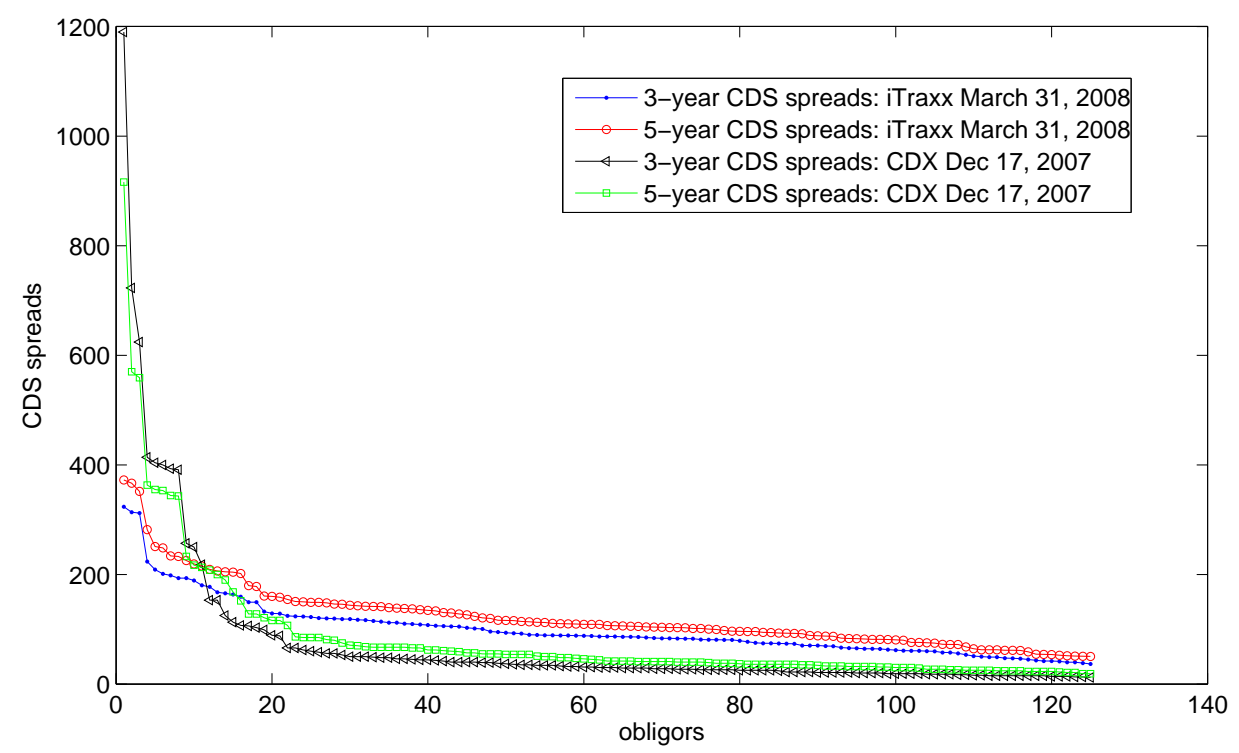

Figure 3: The 3 and 5-year market CDS spreads for the 125 obligors used in the single-name bootstrapping, for the two portfolios CDX.NA.IG sampled on December 17, 2007 and the iTraxx Europe series sampled on March 31, 2008. The CDS spreads are sorted in decreasing order.

Hence, the 125 single-name CDSs constituting the entities in these series are bootstrapped from their market spreads for $T_{1}=3$ and $T_{2}=5$ using piecewise constant individual default intensities on the time intervals $[0,3]$ and $[3,5]$. Figure 3 displays the 3 and 5-year market CDS spreads for the 125 obligors used in the single-name bootstrapping, for the two portfolios CDX.NA.IG sampled on December 17, 2007 and the iTraxx Europe series sampled on March 31, 2008. The CDS spreads are sorted in decreasing order.

When calibrating the joint default intensities $\boldsymbol{\lambda}=\left(\lambda_{I_{j}}^{(k)}\right)_{j, k}$ for the CDX.NA.IG Series 9 , December 17, 2007 we used 5 groups $I_{1}, I_{2}, \ldots, I_{5}$ where $I_{j}=\left\{1, \ldots, i_{j}\right\}$ for $i_{j}=$ $6,19,25,61,125$. Recall that we label the obligors by decreasing level of riskiness. We use the average over 3-year and 5-year CDS spreads as a measure of riskiness. Consequently, obligor 1 has the highest average CDS spread while company 125 has the lowest average CDS spread. Moreover, the obligors in the set $I_{5} \backslash I_{4}$ consisting of the 64 safest companies are assumed to never default individually, and the corresponding CDSs are excluded from the calibration, which in turn relaxes the constraints for $\boldsymbol{\lambda}$ in (48). Hence, the obligors in $I_{5} \backslash I_{4}$ can only bankrupt due to a simultaneous default of the companies in the group $I_{5}=\{1, \ldots, 125\}$, i.e., in an Armageddon event. With this structure the calibration against the December 17, 2007 data-set is very good as can be seen in Table 1. By using stochastic recoveries specified as in (50) and (51) we get a perfect fit of the same data-set. The calibrated common shock intensities $\boldsymbol{\lambda}$ for the 5 groups in the December 17, 2007 data-set, both for constant and stochastic recoveries, are displayed in the left subplot in Figure 4. Note that the shock intensities $\lambda_{I_{j}}^{(1)}$ for the first pillar (i.e. on the interval $[0,3]$ ) follows the same trends both in the constant and stochastic recovery case, while the shock intensities $\lambda_{I_{j}}^{(2)}$ for the second pillar (i.e. on the interval $[3,5]$ ) has less common trend. 
The calibration of the joint default intensities $\boldsymbol{\lambda}=\left(\lambda_{I_{j}}^{(k)}\right)_{j, k}$ for the data sampled at March 31, 2008 is more demanding. This time we use 18 groups $I_{1}, I_{2}, \ldots, I_{18}$ where $I_{j}=\left\{1, \ldots, i_{j}\right\}$ for $i_{j}=1,2, \ldots, 11,13,14,15,19,25,79,125$. In order to improve the fit, as in the 2007-case, we relax the constraints for $\lambda$ in (48) by excluding from the calibration the CDSs corresponding to the obligors in $I_{18} \backslash I_{17}$. Hence, we assume that the obligors in $I_{18} \backslash I_{17}$ never default individually, but can only bankrupt due to an simultaneous default of all companies in the group $I_{18}=\{1, \ldots, 125\}$. In this setting, the calibration of the 2008 data-set with constant recoveries yields an acceptable fit except for the $[3,6]$ tranche, as can be seen in Table 1. However, by including stochastic recoveries (50), (51) the fit is substantially improved as seen in Table 1. Furthermore, in both recovery versions, the more groups added the better the fit, which explain why we use as many as 18 groups.

Table 1: CDX.NA.IG Series 9, December 17, 2007 and iTraxx Europe Series 9, March 31, 2008. The market and model spreads and the corresponding absolute errors, both in bp and in percent of the market spread. The $[0,3]$ spread is quoted in \%. All maturities are for five years.

CDX 2007-12-17: Calibration with constant recovery

\begin{tabular}{c|lllll}
\hline Tranche & {$[0,3]$} & {$[3,7]$} & {$[7,10]$} & {$[10,15]$} & {$[15,30]$} \\
\hline Market spread & 48.07 & 254.0 & 124.0 & 61.00 & 41.00 \\
Model spread & 48.07 & 254.0 & 124.0 & 61.00 & 38.94 \\
Absolute error in bp & 0.010 & 0.000 & 0.000 & 0.000 & 2.061 \\
Relative error in \% & 0.0001 & 0.000 & 0.000 & 0.000 & 5.027 \\
\hline
\end{tabular}

CDX 2007-12-17: Calibration with stochastic recovery

\begin{tabular}{c|lllll}
\hline Tranche & {$[0,3]$} & {$[3,7]$} & {$[7,10]$} & {$[10,15]$} & {$[15,30]$} \\
\hline Market spread & 48.07 & 254.0 & 124.0 & 61.00 & 41.00 \\
Model spread & 48.07 & 254.0 & 124.0 & 61.00 & 41.00 \\
Absolute error in bp & 0.000 & 0.000 & 0.000 & 0.000 & 0.000 \\
Relative error in \% & 0.000 & 0.000 & 0.000 & 0.000 & 0.000 \\
\hline
\end{tabular}

iTraxx Europe 2008-03-31: Calibration with constant recovery

\begin{tabular}{c|lllll}
\hline Tranche & {$[0,3]$} & {$[3,6]$} & {$[6,9]$} & {$[9,12]$} & {$[12,22]$} \\
\hline Market spread & 40.15 & 479.5 & 309.5 & 215.1 & 109.4 \\
Model spread & 41.68 & 429.7 & 309.4 & 215.1 & 103.7 \\
Absolute error in bp & 153.1 & 49.81 & 0.0441 & 0.0331 & 5.711 \\
Relative error in \% & 3.812 & 10.39 & 0.0142 & 0.0154 & 5.218 \\
\hline
\end{tabular}

iTraxx Europe 2008-03-31: Calibration with stochastic recovery

\begin{tabular}{c|lllll}
\hline Tranche & {$[0,3]$} & {$[3,6]$} & {$[6,9]$} & {$[9,12]$} & {$[12,22]$} \\
\hline Market spread & 40.15 & 479.5 & 309.5 & 215.1 & 109.4 \\
Model spread & 40.54 & 463.6 & 307.8 & 215.7 & 108.3 \\
Absolute error in bp & 39.69 & 15.90 & 1.676 & 0.5905 & 1.153 \\
Relative error in \% & 0.9886 & 3.316 & 0.5414 & 0.2745 & 1.053 \\
\hline
\end{tabular}


Common shock intensities for CDX.NA.IG, 2007-12-17

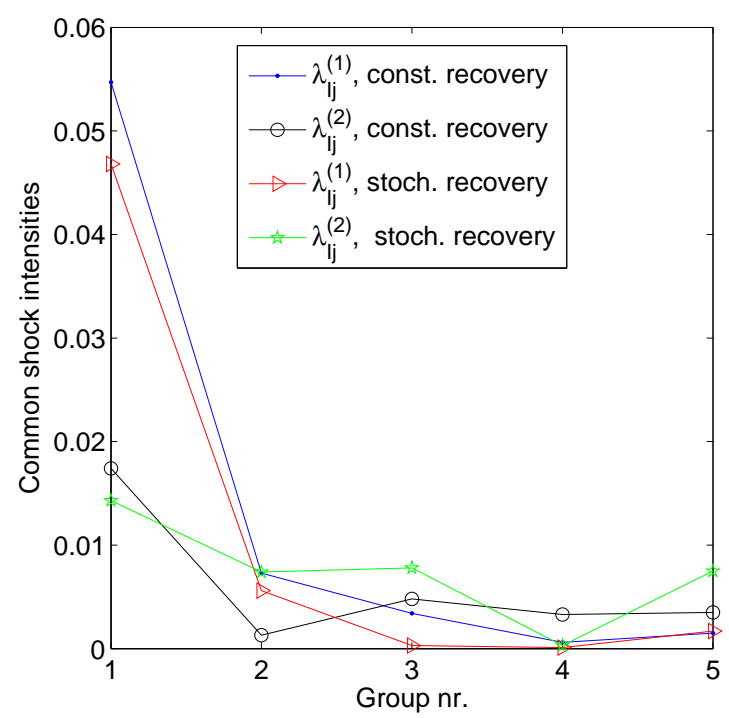

Common shock intensities for iTraxx Europe, 2008-03-31

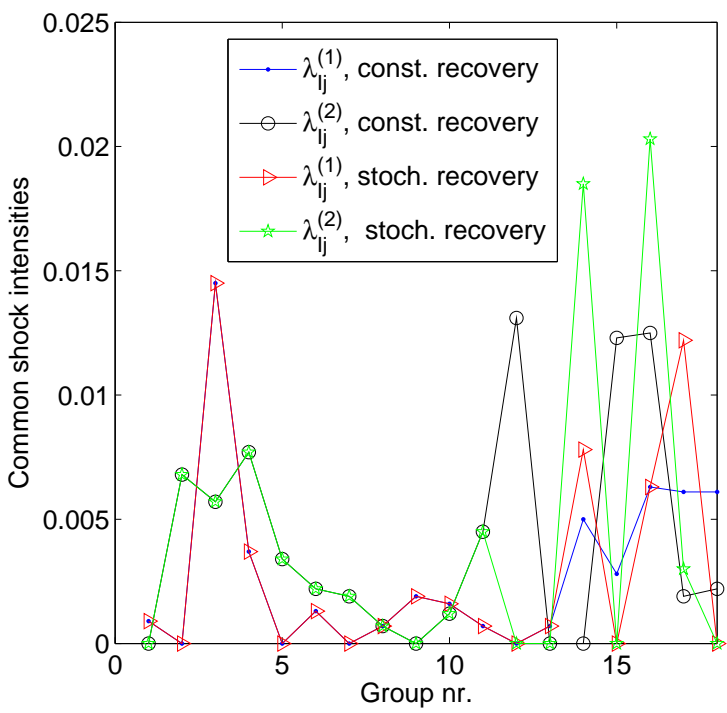

Figure 4: The calibrated common shock intensities $\left(\lambda_{I_{j}}^{(k)}\right)_{j, k}$ both in the constant and stochastic recovery case for the two portfolios CDX.NA.IG sampled on December 17, 2007 (left) and the iTraxx Europe series sampled on March 31, 2008 (right).

The calibrated common shock intensities $\boldsymbol{\lambda}$ for the 18 groups in the March 2008 dataset, both for constant and stochastic recoveries, are displayed in the right subplot in Figure 4 . In this subplot we note that for the 13 first groups $I_{1}, \ldots, I_{13}$, the common shock intensities $\lambda_{I_{j}}^{(1)}$ for the first pillar are identical in the constant and stochastic recovery case, and then diverge quite a lot on the last five groups $I_{14}, \ldots, I_{18}$, except for group $I_{16}$. Similarly, in the same subplot we also see that for the 11 first groups $I_{1}, \ldots, I_{11}$, the shock intensities $\lambda_{I_{j}}^{(2)}$ for the second pillar are identical in the constant and stochastic recovery case, and then differ quite a lot on the last seven groups, except for group $I_{13}$. The optimal parameters $q$ and $p_{0}$ used in the stochastic recovery model was given by $q=0.4405$ and $p_{0}=0.4$ for the 2007 data set and $q=0.6002$ and $p_{0}=0.4$ for the 2008 case.

Let us finally discuss the choice of the groupings $I_{1} \subset I_{2} \subset \ldots \subset I_{m}$ in our calibrations. First, for the CDX.NA.IG Series 9, December 17, 2007 data set, we used $m=5$ groups with as always $i_{m}=n$. For $j=1,2$ and 4 the choice of $i_{j}$ corresponds to the number of defaults needed for the loss process with constant recovery of $40 \%$ to reach the $j$-th attachment points. Hence, $i_{j} \cdot \frac{1-R}{n}$ with $R=40 \%$ and $n=125$ then approximates the attachment points $3 \%, 10 \%, 30 \%$ which explains the choice $i_{1}=6, i_{2}=19, i_{4}=61$. The choice of $i_{3}=25$ implies a loss of $12 \%$ and gave a better fit than choosing $i_{3}$ to exactly match $15 \%$. Finally, no group was chosen to match the attachment point of $7 \%$ since this made the calibration worse off for all groupings we tried. With the above grouping structure we got almost perfect fits in the constant recovery case, and perfect fit with stochastic recovery, as was seen in Table 1. Unfortunately, using the same technique on the market CDO data from the iTraxx Europe series sampled on March 31, 2008 was not enough to achieve good calibrations. Instead more groups had to be added and we tried different groupings which led to the optimal choice rendering the calibration in Table 1. To this end, it is of interest to study the sensitivity of the calibrations with respect to the choice of the 
groupings on the form $I_{1} \subset I_{2} \subset \ldots \subset I_{m}$ where $I_{j}=\left\{1, \ldots, i_{j}\right\}$ for $i_{j} \in\{1,2, \ldots, m\}$ and $i_{1}<\ldots<i_{m}=125$ on the March 31, 2008, data set. Three such groupings are displayed in Table 2 and the corresponding calibration results on the 2008 data set is showed in Table 3.

Table 2: Three different groupings (denoted A,B and C) consisting of $m=7,9,13$ groups having the structure $I_{1} \subset I_{2} \subset \ldots \subset I_{m}$ where $I_{j}=\left\{1, \ldots, i_{j}\right\}$ for $i_{j} \in\{1,2, \ldots, m\}$ and $i_{1}<\ldots<i_{m}=125$.

Three different groupings

\begin{tabular}{r|lllllllllllll}
\hline$i_{j}$ & $i_{1}$ & $i_{2}$ & $i_{3}$ & $i_{4}$ & $i_{5}$ & $i_{6}$ & $i_{7}$ & $i_{8}$ & $i_{9}$ & $i_{10}$ & $i_{11}$ & $i_{12}$ & $i_{13}$ \\
\hline Grouping A & 6 & 14 & 15 & 19 & 25 & 79 & 125 & & & & & & \\
Grouping B & 2 & 4 & 6 & 14 & 15 & 19 & 25 & 79 & 125 & & & & \\
Grouping C & 2 & 4 & 6 & 8 & 9 & 10 & 11 & 14 & 15 & 19 & 25 & 79 & 125 \\
\hline
\end{tabular}

Table 3: The relative calibration error in percent of the market spread, for the three different groupings A, B and C in Table 2, when calibrated against CDO tranche on iTraxx Europe Series 9, March 31, 2008 (see also in Table 1).

\begin{tabular}{r|ccccc}
\multicolumn{6}{c}{ Relative calibration error in \% (constant recovery) } \\
\hline Tranche & {$[0,3]$} & {$[3,6]$} & {$[6,9]$} & {$[9,12]$} & {$[12,22]$} \\
\hline Error for grouping A & 6.875 & 18.33 & 0.0606 & 0.0235 & 4.8411 \\
Error for grouping B & 6.622 & 16.05 & 0.0499 & 0.0206 & 5.5676 \\
Error for grouping C & 4.107 & 11.76 & 0.0458 & 0.0319 & 3.3076 \\
\hline
\end{tabular}

Relative calibration error in \% (stochastic recovery)

\begin{tabular}{r|lllll}
\hline Tranche & {$[0,3]$} & {$[3,6]$} & {$[6,9]$} & {$[9,12]$} & {$[12,22]$} \\
\hline Error for grouping A & 3.929 & 9.174 & 2.902 & 1.053 & 2.109 \\
Error for grouping B & 2.962 & 7.381 & 2.807 & 1.002 & 1.982 \\
Error for grouping C & 1.439 & 4.402 & 0.5094 & 0.2907 & 1.235 \\
\hline
\end{tabular}

From Table 3 we see that in the case with constant recovery the relative calibration error in percent of the market spread decreased monotonically for the first three thranches as the number of groups increased. Furthermore, in the case with stochastic recovery the relative calibration error decreased monotonically for all five tranches as the number of groups increased in each grouping. The rest of the parameters in the calibration where the same as in the optimal calibration in Table 1.

Finally, we remark that the two optimal groupings used in Table 1 in the two different data sets CDX.NA.IG Series 9, December 17, 2007 and iTraxx Europe Series 9, March 31, 2008 differ quite a lot. However, the CDX.NA.IG Series is composed by North American obligors while the iTraxx Europe Series is formed by European companies. Thus, there is no model risk or inconsistency created by using different groupings for these two different data sets, coming from two disjoint markets. If on the other hand the same series is calibrated and assessed (e.g. for hedging) at different time points in a short time span, it is of course desirable to use the same grouping in order to avoid model risk. 


\subsubsection{The Implied Loss Distribution}

After the fit of the model against market spreads we can use the calibrated portfolio parameters $\boldsymbol{\lambda}=\left(\lambda_{I_{j}}^{(k)}\right)_{j, k}$ together with the calibrated individual default intensities, to study the credit-loss distribution in the portfolio. In this paper we only focus on some few examples derived from the loss distribution with constant recoveries evaluated at $T=5$ years.
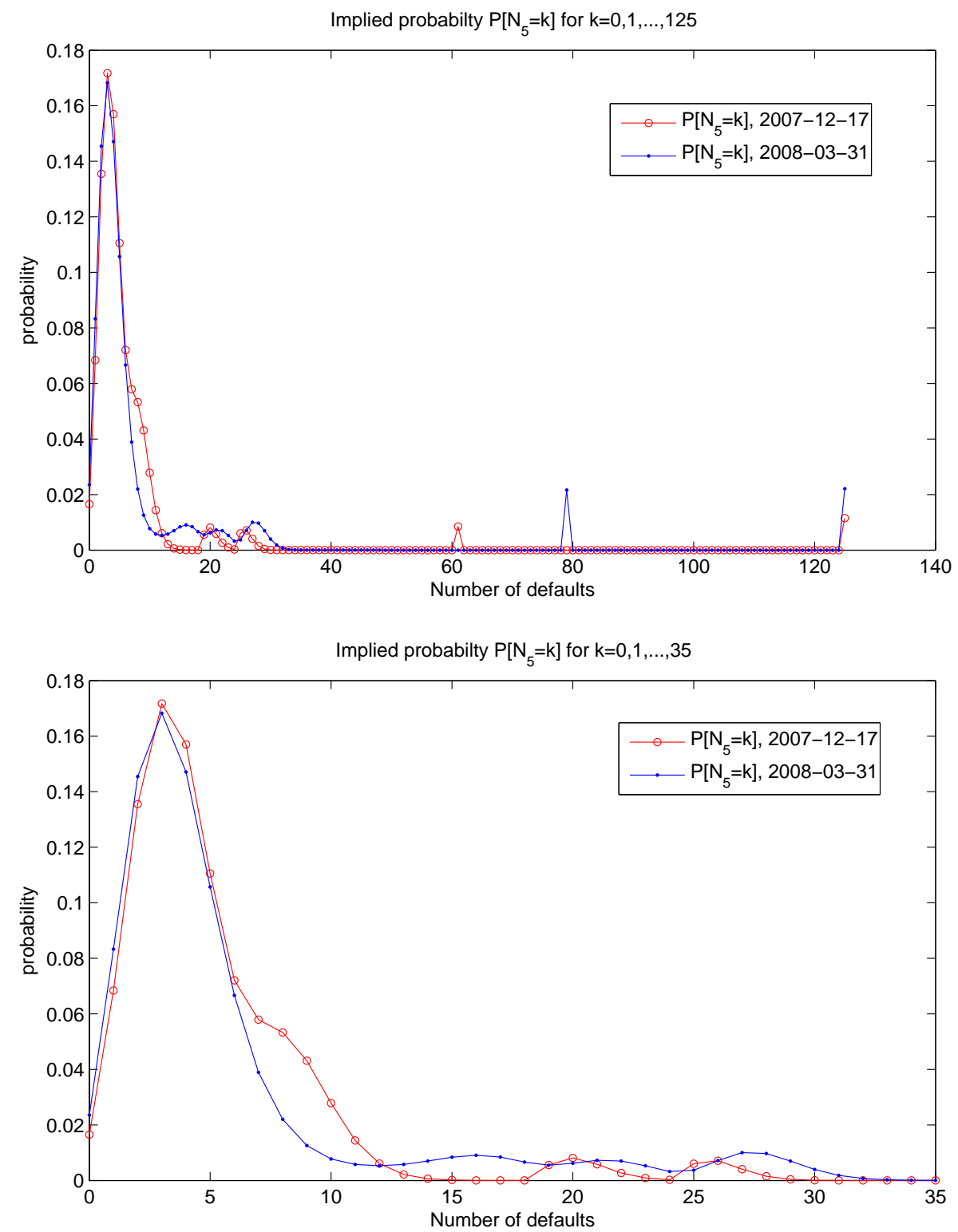

Figure 5: The implied distribution $\mathbb{P}\left[N_{5}=k\right]$ on $\{0,1, \ldots, \ell\}$ where $\ell=125$ (top) and $\ell=35$ (bottom) when the model is calibrated against CDX.NA.IG Series 9, December 17, 2007 and iTraxx Europe Series 9, March 31, 2008.

The allowance of joint defaults of the obligors in the groups $I_{j}$ together with the restriction of the most safest obligors not being able to default individually, will lead to 
some interesting effects of the loss distribution, as can be seen in Figures 5 and 6 . For example, we clearly see that the support of the loss-distributions will in practice be limited to a rather compact set. To be more specific, the upper and lower graphs in Figure 5 indicate that $\mathbb{P}\left[N_{5}=k\right]$ roughly has support on the set $\{1, \ldots, 35\} \cup\{61\} \cup\{125\}$ for the 2007 case and on $\{1, \ldots, 40\} \cup\{79\} \cup\{125\}$ for the 2008 data-set. This becomes even more clear in a log-loss distribution, as is seen in the upper and lower graphs in Figure 6.
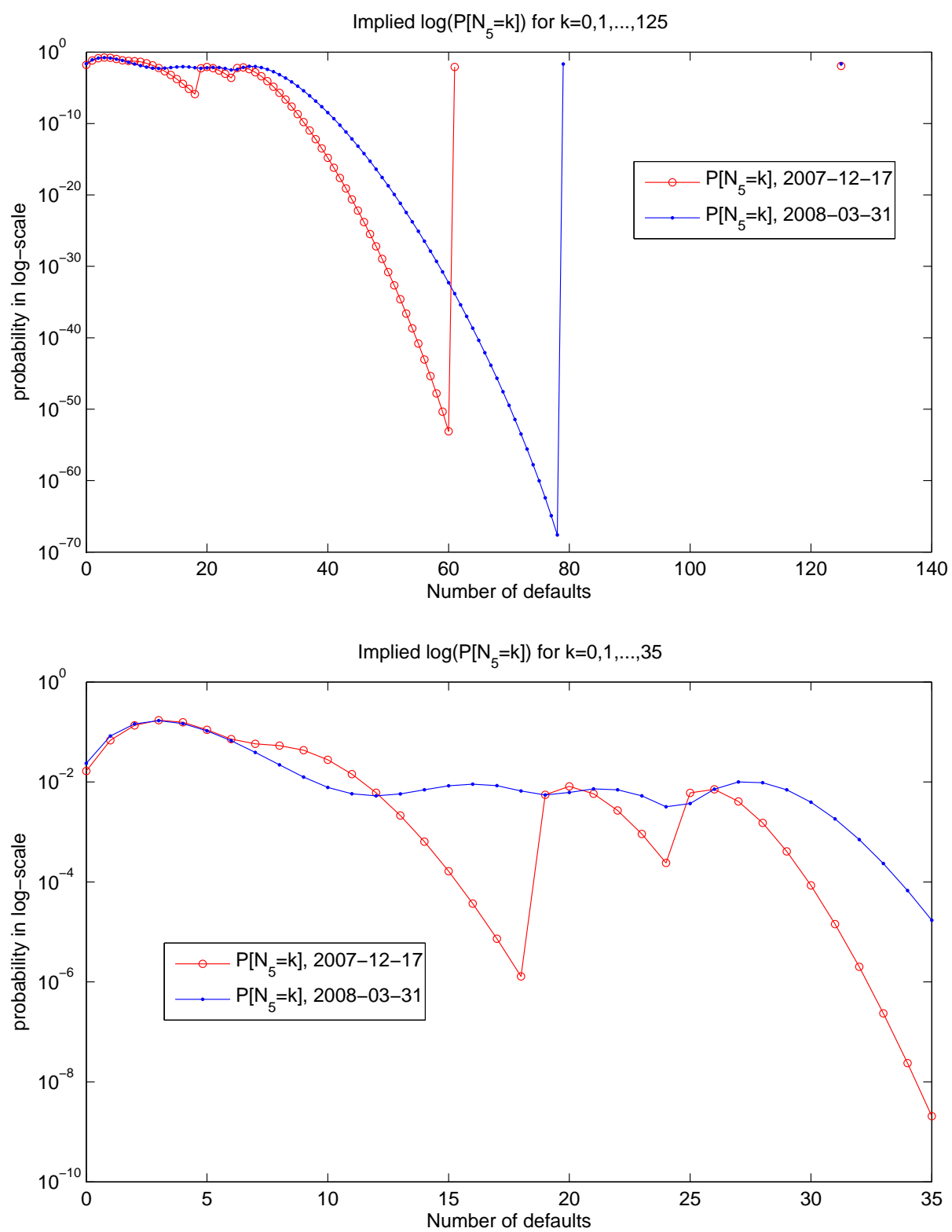

Figure 6: The implied $\log$ distribution $\ln \left(\mathbb{P}\left[N_{5}=k\right]\right)$ on $\{0,1, \ldots, \ell\}$ where $\ell=125$ (top) and $\ell=35$ (bottom) when the model is calibrated against CDX.NA.IG Series 9, December 17, 2007 and iTraxx Europe Series 9, March 31, 2008.

From the upper graph in Figure 6 we see that the default-distribution is nonzero on 
$\{36, \ldots, 61\}$ in the 2007-case and nonzero on $\{41, \ldots, 79\}$ for the 2008 -sample, but the actual size of the loss-probabilities are in the range $10^{-10}$ to $10^{-70}$. Such low values will obviously be treated as zero in any practically relevant computation. Furthermore, the reasons for the empty gap in the upper graph in Figure 6 on the interval $\{62, \ldots, 124\}$ for the 2007-case is due to the fact that we forced the obligors in the set $I_{5} \backslash I_{4}$ to never default individually, but only due to an simultaneous common shock default of the companies in the group $I_{5}=\{1, \ldots, 125\}$. This Armageddon event is displayed as an isolated nonzero 'dot' at default nr 125 in the upper graph of Figure 6. The gap on $\{80, \ldots, 124\}$ in the 2008 case is explained similarly due to our assumption on the companies in the set $I_{19} \backslash I_{18}$. Also note that the two 'dots' at default nr 125 in the top plot of Figure 6 are manifested as spikes in the upper graph displayed in Figure 5. The shape of the multimodal loss distributions presented in Figure 5 and Figure 6 are typical for models allowing simultaneous defaults, see for example Figure 2, page 59 in [13] and Figure 2, page 710 in [22].

\subsection{Calibration Methodology and Results with Stochastic Intensities}

We now consider that pre-specified group intensities are stochastic CIR processes given as in Example 2.7. For simplicity, $a$ and $c$ are fixed a priori and we assume a piecewise-constant parameterization of every mean-reversion function $b_{Y}(t)$ (see expression (13)), so for every $k=1 \ldots M$,

$$
b_{Y}(t)=b_{Y}^{(k)}, t \in\left[T_{k-1}, T_{k}\right)
$$

where $T_{0}=0$. The time grid $\left(T_{k}\right)$ is the same than the one used in the previous section, i.e., $M=2, T_{1}=3, T_{2}=5$. It corresponds to the set of standard CDS maturities which are lower or equal to the maturity of the fitted CDO tranches. In order to reduce the number of parameters at hands, we consider that, for every group $Y \in \mathcal{Y}$, the starting point of the corresponding intensity process is given by its first-pillar mean-reversion parameter, i.e., $X_{0}^{Y}=b_{Y}^{(1)}$. This specification guarantees that there are exactly the same number of parameters to fit than in Subsection 4.1 (piecewise-constant intensities and constant recovery). All other aspects of the model are the same as in Subsection 4.1. So, we reproduce the same calibration methodology except that now individual mean-reversion parameters $\left\{b_{i}^{(k)}: i=1, \ldots, n\right.$ and $\left.k=1,2\right\}$ play the role of former parameters $\left\{\lambda_{i}^{(k)}: i=\right.$ $1, \ldots, n$ and $k=1,2\}$ and shock parameters $\left\{b_{I_{j}}^{(k)}: j=1, \ldots, m\right.$ and $\left.k=1,2\right\}$ play the role of former parameters $\left\{\lambda_{I_{j}}^{(k)}: j=1, \ldots, m\right.$ and $\left.k=1,2\right\}$.

In order to construct a tractable CDS and CDO pricer, the "building blocks" survival group probabilities

$$
\mathbb{E}\left[\exp \left(-\int_{0}^{t} X_{u}^{Y} d u\right)\right]
$$

have to be computed very efficiently. Fortunately, for CIR processes $X^{Y}$, the latter quantities are solution of related ODEs which can be solved analytically.

We now study the calibration performance of this model specification. Playing with different values of parameters $a$ (speed of mean-reversion) and $c$ (volatility) may slightly affect the quality of the fit. We consider thereafter that $a=3$ and $c=0.5$ which render our best results. 
Table 4: CDX.NA.IG Series 9, December 17, 2007. The market and model spreads and the corresponding absolute errors, both in bp and in percent of the market spread. The $[0,3]$ spread is quoted in \%. All maturities are for five years.

CDX 2007-12-17: Calibration with constant recovery

\begin{tabular}{c|lllll}
\hline Tranche & {$[0,3]$} & {$[3,7]$} & {$[7,10]$} & {$[10,15]$} & {$[15,30]$} \\
\hline Market spread & 48.07 & 254.0 & 124.0 & 61.00 & 41.00 \\
Model spread & 50.37 & 258.01 & 124.68 & 61.32 & 41.91 \\
Absolute error in bp & 2.301 & 4.016 & 0.684 & 0.327 & 0.912 \\
Relative error in \% & 4.787 & 1.581 & 0.552 & 0.536 & 2.225 \\
\hline
\end{tabular}

As can be seen in Table 4, we obtain a correct fit for CDX 2007-12-17 even in the case where no name is removed from the calibration constraints. Here, we use 5 groups $I_{1}, I_{2}, \ldots, I_{5}$ where $I_{j}=\left\{1, \ldots, i_{j}\right\}$ for $i_{j}=8,19,27,102,125$. Note that when the last group $I_{5}$ is retrieved from the calibration constraints, we are able to obtain a perfect fit as observed with the piecewise constant intensity model with stochastic recovery.

For iTraxx Europe 2008-03-31, the calibration results are not improved with respect to the piecewise-constant intensity model with constant recovery.

\subsection{Min-Variance Hedging Deltas}

In this subsection we present some numerical results illustrating performance of the minvariance hedging strategies given in Proposition 3.2. We here only focus on hedging strategies for the data of CDX.NA.IG Series 9 on December 17, 2007 calibrated in the constant recovery model presented in Subsection 4.1 ,

The aim of this subsection is to analyze the composition of the hedging portfolio at time $t=0$ (the calibration date) when standardized CDO tranches are hedged with a group of $d$ single-name CDSs, which are included in the underlying CDS index. Since no spread factor $\mathbf{X}$ is used in the model, Proposition 3.2 then implies that the min-variance hedging ratios at time $t=0$ is given by $\zeta^{v a}\left(0, \mathbf{H}_{0}\right)=(u, \mathbf{v})(\mathbf{v}, \mathbf{v})^{-1}\left(0, \mathbf{H}_{0}\right)$ where

$$
(u, \mathbf{v})=\sum_{Y \in \mathcal{Y}} \lambda_{Y}(0) \Delta u^{Y}\left(\Delta \mathbf{v}^{Y}\right)^{\top} \quad \text { and } \quad(\mathbf{v}, \mathbf{v})=\sum_{Y \in \mathcal{Y}} \lambda_{Y}(0) \Delta \mathbf{v}^{Y}\left(\Delta \mathbf{v}^{Y}\right)^{\top} .
$$

Hence, computing the min-variance hedging ratios involves a summation of the "jump differentials" $\lambda_{Y}(0) \Delta u^{Y}\left(\Delta \mathbf{v}^{Y}\right)^{\top}$ and $\lambda_{Y}(0) \Delta \mathbf{v}^{Y}\left(\Delta \mathbf{v}^{Y}\right)^{\top}$ over all possible triggering events $Y \in \mathcal{Y}$ where $\mathcal{Y}=\left\{\{1\}, \ldots,\{n\}, I_{1}, \ldots, I_{m}\right\}$.

In the calibration of the CDX.NA.IG Series 9 , we used $m=5$ groups $I_{1}, I_{2}, \ldots, I_{5}$ where $I_{j}=\left\{1, \ldots, i_{j}\right\}$ for $i_{j}=6,19,25,61,125$ and the obligors have been labeled by decreasing level of riskiness. At the calibration date $t=0$ associated with December 17, 2007, no name has defaulted in CDX Series 9 so we set $\mathbf{H}_{0}=\mathbf{0}$. In our empirical framework, the intensities $\lambda_{Y}(0), Y \in \mathcal{Y}$ are computed from the constant default intensities $\lambda_{i}$ that fit market spreads of 3-year maturity CDSs and from the 3-year horizon joint default intensities $\lambda_{I_{j}}$ calibrated to CDO tranche quotes. The terms $\Delta u^{Y}\left(0, \mathbf{H}_{0}\right)$ and $\Delta \mathbf{v}^{Y}\left(0, \mathbf{H}_{0}\right)$ corresponds to the change in value of the tranche and the single-name CDSs, at the arrival of the triggering event affecting all names in group $Y$. Recall that the cumulative change in value of the tranche is equal to

$$
\Delta u^{Y}\left(0, \mathbf{H}_{0}\right)=L_{a, b}\left(\mathbf{H}_{0}^{Y}\right)-L_{a, b}\left(\mathbf{H}_{0}\right)+u\left(0, \mathbf{H}_{0}^{Y}\right)-u\left(0, \mathbf{H}_{0}\right)
$$


where $\mathbf{H}_{0}^{Y}$ is the vector of $\{0,1\}^{n}$ such that only the components $i \in Y$ are equal to one. Hence, the tranche sensitivity $\Delta u^{Y}\left(0, \mathbf{H}_{0}\right)$ includes both the protection payment on the tranche associated with the default of group $Y$ and the change in the ex-dividend price $u$ of the tranche. Note that the price sensitivity is obtained by computing the change in the present value of the default leg and the premium leg. The latter quantity involves the contractual spread that defines cash-flows on the premium leg. As for CDX.NA.IG Series 9, the contractual spreads were equal to $500 \mathrm{bps}, 130 \mathrm{bps}, 45 \mathrm{bps}, 25 \mathrm{bps}$ and 15 bps for the tranches [0-3\%], [3-7\%], [7-10\%], [10-15\%] and [15-30\%]. We use the common-shock interpretation to compute $u\left(0, \mathbf{H}_{0}^{Y}\right)$ and $u\left(0, \mathbf{H}_{0}\right)$ with the convolution recursion pricing scheme detailed in Subsection 3.3. More precisely, using the same notation as in Subsection 3.3, the CDO tranche price $u\left(0, \mathbf{H}_{0}^{Y}\right)$ (resp. $u\left(0, \mathbf{H}_{0}\right)$ ) is computed using the recursion procedure with $Z=\mathbb{N}_{n} \backslash Y$ (resp. $Z=\mathbb{N}_{n}$ ). We let $i_{1}, \ldots i_{d}$ be the CDSs used in the min-variance hedging and assume that they all are initiated at time $t=0$. Hence, the market value at $t=0$ for these CDSs are zero. As a result, when group $Y$ defaults simultaneously, the change in value $\Delta \mathbf{v}^{Y}\left(0, \mathbf{H}_{0}\right)$ for buy-protection positions on these CDSs is only due to protection payment associated with names in group $Y$. Hence, for one unit of nominal exposure on hedging CDSs, the corresponding vector of sensitivities is equal to $\Delta \mathbf{v}^{Y}\left(0, \mathbf{H}_{0}\right)=\left((1-R) \mathbb{1}_{i_{1} \in Y}, \ldots,(1-R) \mathbb{1}_{i_{d} \in Y}\right)^{\top}$ where the recovery rate $R$ is assumed to be constant and equal to $40 \%$.

Table 5: The names and CDS spreads (in bp) of the six riskiest obligors used in the hedging strategy displayed by Figure 7 .

\begin{tabular}{l|cccccc} 
Company (Ticker) & CCR-HomeLoans & RDN & LEN & SFI & PHM & CTX \\
\hline 3-year CDS spread & 1190 & 723 & 624 & 414 & 404 & 393
\end{tabular}

Figure 7 displays the nominal exposure for the $d$ most riskiest CDSs when hedging one unit of nominal exposure in a CDO by using the min-variance hedging strategy in Proposition 3.2. We use $d=3,4,5$ and $d=6$ in our computations. Furthermore, Table 5 displays the names and sizes of the 3-year CDS spreads used in the hedging strategy. Each plot in Figure 7 should be interpreted as follows: in every pair $(x, y)$ the $x$-component represents the size of the 3 -year CDS spread at the hedging time $t=0$ while the $y$-component is the corresponding nominal CDS-exposure computed via Proposition 3.2 using the $d$ riskiest CDSs. The graphs are ordered from top to bottom, where the top panel corresponds to hedging with the $d=3$ riskiest CDS and the bottom panel corresponds to hedging with the $d=6$ riskiest names. Note that the $x$-axes are displayed from the riskiest obligor to the safest. Thus, hedge-sizes $y$ for riskier CDSs are aligned to the left in each plot while $y$-values for safer CDSs are consequently displayed more to the right. In doing this, going from the top to the bottom panel consists in observing the effect of including new safer names from the right part of the graphs. We have connected the pairs $(x, y)$ with lines forming graphs that visualizes possible trends of the min-variance hedging strategies for the $d$ most riskiest CDSs. For example, when the three riskiest names are used for hedging (top panel), we observe that the amount of nominal exposure in hedging instruments decreases with the degree of subordination, i.e., the [0-3\%] equity tranche requires more nominal exposure in CDSs than the upper tranches. 

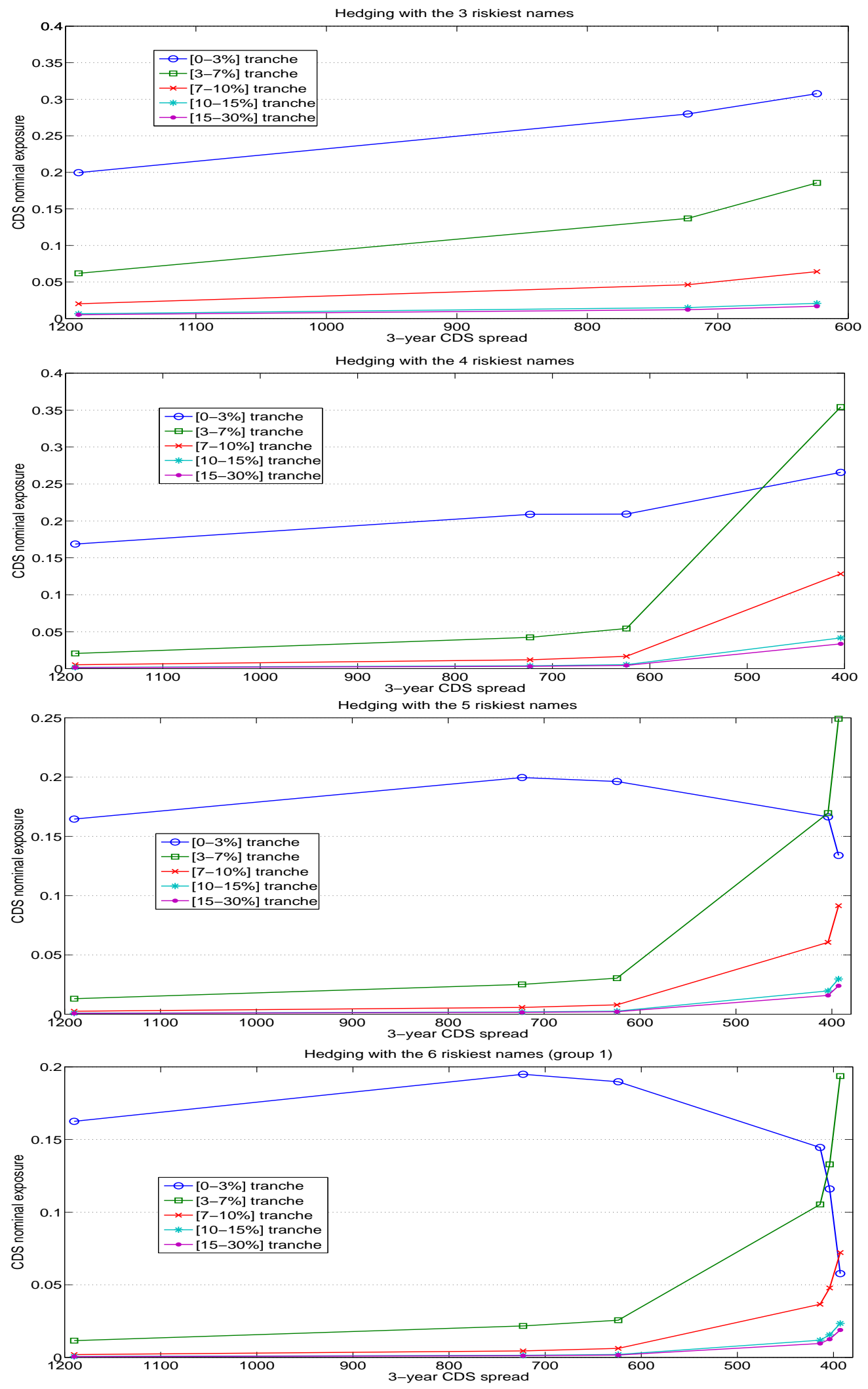

Figure 7: Min-variance hedging strategies associated with the $d$ riskiest CDSs, $d=3,4,5,6$ for one unit of nominal exposure of different CDO tranches in a model calibrated to market spreads of CDX.NA.IG Series 9 on December 17, 2007. 
Note moreover that the min-variance hedging portfolio contains more CDSs on names with lower spreads. When lower-spread CDSs are added in the portfolio, the picture remains almost the same for the 3 riskiest names. For the remaining safer names however, the picture depends on the characteristics of the tranche. For the [0-3\%] equity tranche, the quantity of the remaining CDSs required for hedging sharply decrease as additional safer names are added. One possible explanation is that adding too many names in the hedging strategy will be useless when hedging the equity tranche. This is intuitively clear since one expects that the most riskiest obligors will default first and consequently reduce the equity tranche substantially, explaining the higher hedge-ratios for riskier names, while it is less likely that the more safer names will default first and thus incur losses on the first tranche which explains the lower hedge ratios for the safer names. We observe the opposite trend for the senior (safer) tranches: adding new (safer) names in the hedging portfolio seems to be useful for "non equity" tranches since the nominal exposure required for these names increases when they are successively added.

Figure 8 and 9 display min-variance hedging strategies when hedging a standard tranche with the 61 riskiest names, i.e., all names excepted names in group $I_{5} \backslash I_{4}$. Contrary to Figure 7 , these graphs allow to visualize the effect of the "grouping structure" on the composition of the hedging portfolio. In this respect, we use different marker styles in order to distinguish names in the different disjoint groups $I_{1}, I_{2} \backslash I_{1}, I_{3} \backslash I_{2}, I_{4} \backslash I_{3}$. As one can see, the min-variance hedging strategies are quite different among tranches. Moreover, whereas nominal exposures required for hedging are clearly monotone for names belonging to the same disjoint group, this tendency is broken when we consider names in different groups. This suggests that the grouping structure has a substantial impact on the distribution of names in the hedging portfolio. For the equity tranche, we observe in Figure 7 that less safer-names are required for hedging. This feature is retained in Figure 8 when we look at names in specific disjoint groups. Indeed, names in a given disjoint group are affected by the same common-shocks which in turn affect the equity tranche with the same severity. The only effect that may explain differences in nominal exposure among names in the same disjoint group is spontaneous defaults: names with wider spreads are more likely to default first, then we need them in greater quantity for hedging than names with tighter spreads.

Note that nominal exposure in hedging CDS even becomes negative for names within groups $I_{2} \backslash I_{1}$ and $I_{4} \backslash I_{3}$ when spreads are low. However, in Figure 8 we observe that, for the equity tranche, some of the riskiest names in $I_{4} \backslash I_{3}$ are more useful in the hedging than some of the safest names in group $I_{1}$, which may sound strange at a first glance, given that the credit spread of the latter is much larger than the credit spread of the former. Recall that the equity tranche triggers protection payments corresponding to the few first defaults, if these occur before maturity. Even if names in group $I_{4} \backslash I_{3}$ have a very low default probability, the fact that they can affect the tranche at the arrival of common-shocks $I_{4}$ or $I_{5}$ makes these names appealing for hedging because they are less costly (they require less premium payments) than names in $I_{1}$.

Figure 8 suggests that names with the lowest spreads should be ineffective to hedge the $[0-3 \%]$ and the $[3-7 \%]$ tranches. As can be seen in Figure 9, this is the contrary for the other tranches, i.e., the amount of low-spread names in the hedging portfolio increases as the tranche becomes less and less risky. For the [15-30\%] super-senior tranche, we can see on the lowest graph of Figure 9 that the safer a name is, the larger the quantity which is required for hedging. Furthermore, Figure 9 also shows that in a consistent dynamic model of portfolio credit risk calibrated to a real data set, the [15-30\%] super-senior tranche has significant (in fact, most of its) sensitivity to very safe names with spreads less than a few 
dozens of bp-s. For this tranche it is actually likely that one could improve the hedge by inclusion of even safer names to the set of hedging instruments, provided these additional names could also be calibrated to. Recall that on the data of CDX.NA.IG Series 9 on December 17, 2007, we calibrated our model to the 64 safest names in the portfolio.
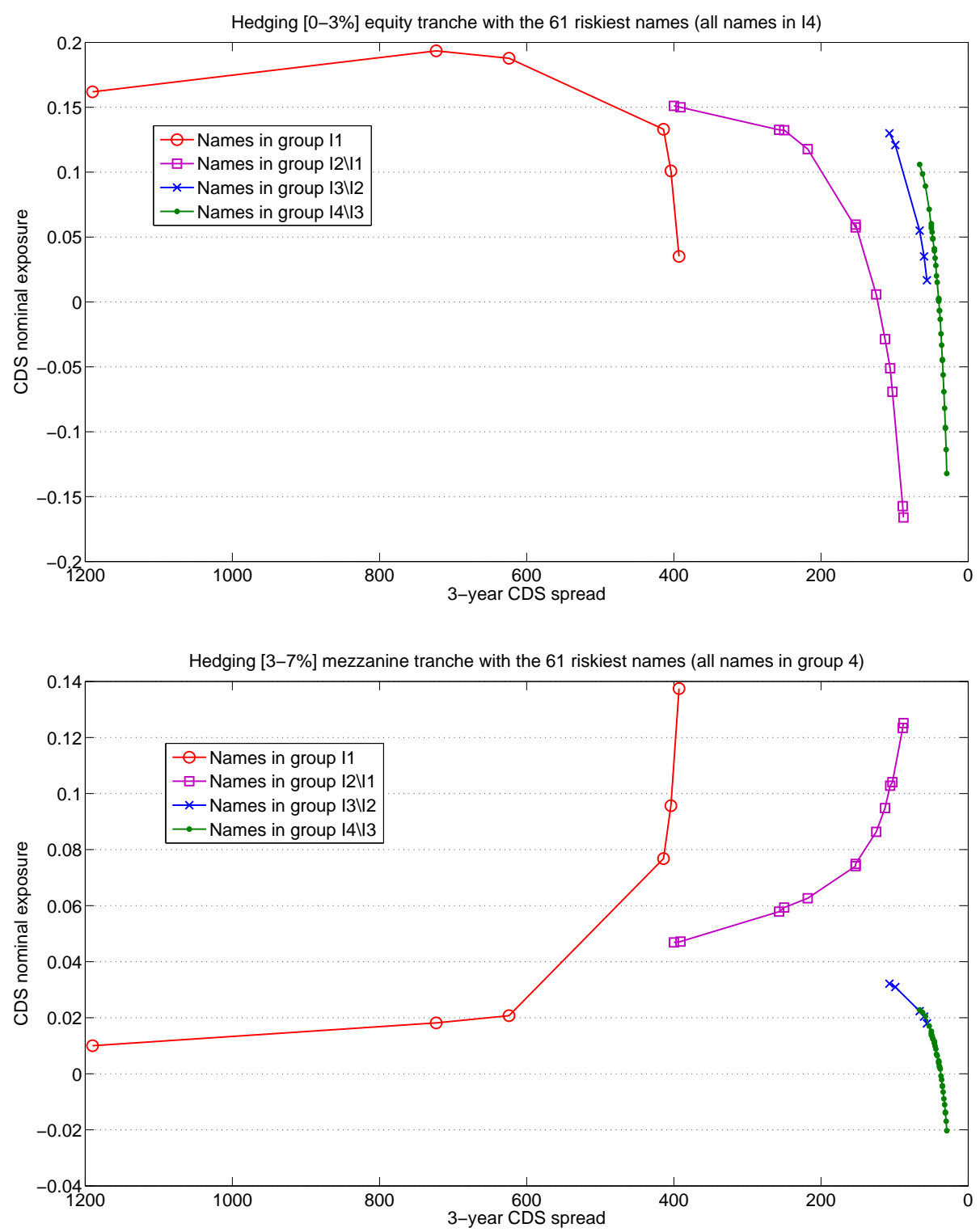

Figure 8: Min-variance hedging strategies when hedging one unit of nominal exposure in the $[0-3 \%]$ equity tranche (top) and the [3-7\%] mezzanine tranche (bottom) using the $d$ riskiest CDSs, $d=61$ (all names excepted names in group $I_{5} \backslash I_{4}$ ) for one unit of nominal exposure. 

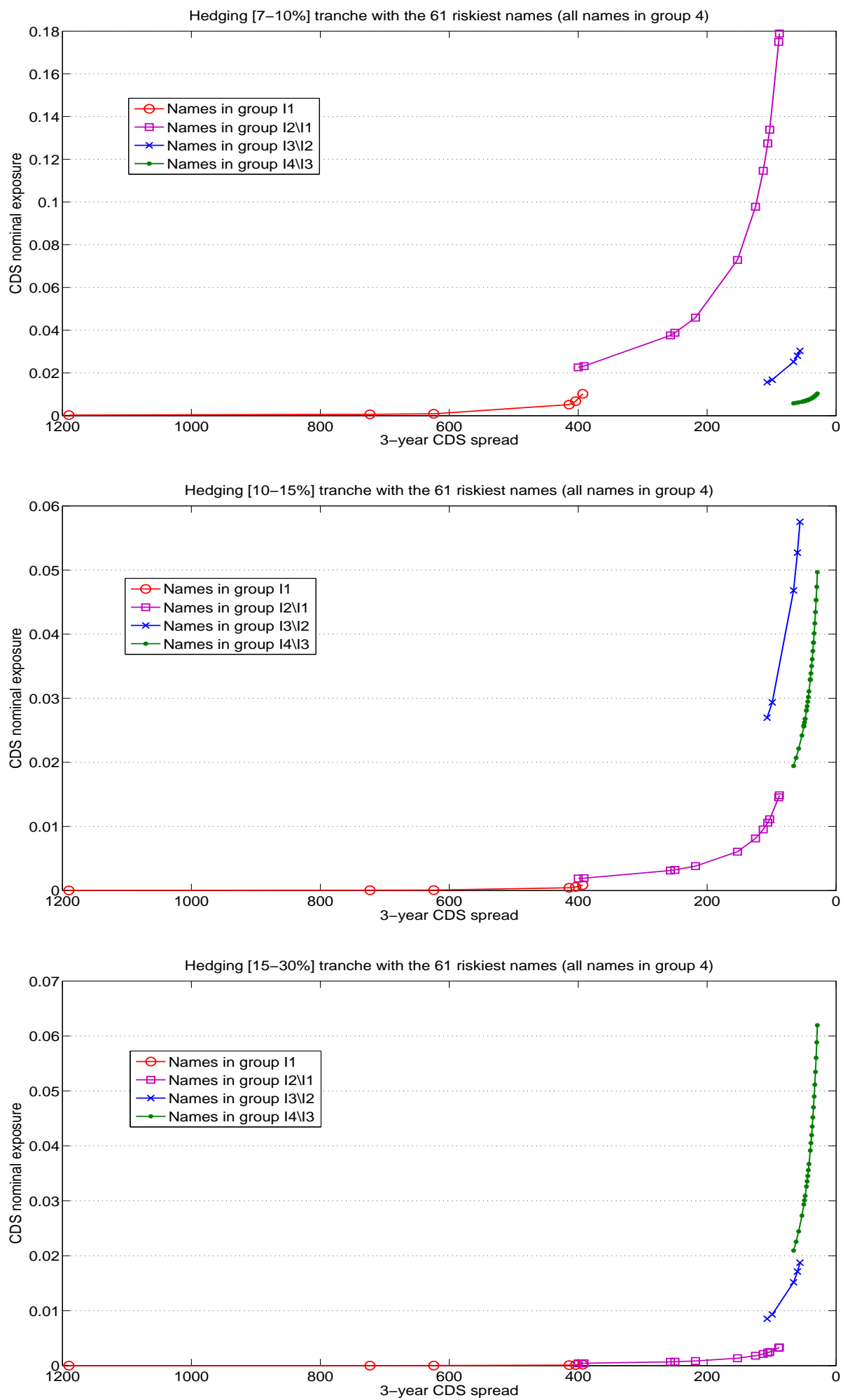

Figure 9: Min-variance hedging strategies when hedging one unit of nominal exposure in the $[7-10 \%]$ tranche (top), the [10-15\%] tranche (middle) and the [15-30\%] tranche (bottom) with the $d$ riskiest CDSs, $d=61$ (all names excepted names in group $I_{5} \backslash I_{4}$ ). 


\section{A Appendix}

\section{A.1 Model Construction}

The point process $\mathbf{H}$ with intensity depending on the factor process $\mathbf{X}$ in (3), is constructed by an $\mathbf{X}$-related change of probability measure, starting from an independent continuoustime Markov chain. So, given a factor process $\mathbf{X}$ as in (1), let $\mathbf{H}$ denote a continuous-time Markov chain with $\widehat{\mathbb{P}}$-intensity one of transition from $\mathbf{k}$ to $\mathbf{l}$, for every $\mathbf{l} \neq \mathbf{k}$. Let then the $\widehat{\mathbb{P}}$-martingal 99 be defined by $\Gamma_{0}=1$ and, for $t \in[0, T]$,

$$
\begin{aligned}
\frac{d \Gamma_{t}}{\Gamma_{t-}} & =\sum_{l \in\{0,1\}^{n}}\left(\lambda\left(t, \mathbf{X}_{t}, \mathbf{H}_{t-}, \mathbf{l}\right)-1\right)\left(d N_{t}\left(\mathbf{H}_{t-}, \mathbf{l}\right)-\mathbb{1}_{\mathbf{l} \neq \mathbf{H}_{t-}} d t\right) \\
& =\sum_{\mathbf{l} \neq \mathbf{H}_{t-}}\left(\lambda\left(t, \mathbf{X}_{t}, \mathbf{H}_{t-}, \mathbf{l}\right)-1\right)\left(d N_{t}\left(\mathbf{H}_{t-}, \mathbf{l}\right)-d t\right),
\end{aligned}
$$

where the functions $\lambda(t, \mathbf{x}, \mathbf{k}, \mathbf{l})$ are those of (3)), and where $N_{t}(\mathbf{k}, \mathbf{l})$ is the point process with $\widehat{\mathbb{P}}$-intensity $\mathbb{1}_{\left\{\mathbf{k}=\mathbf{H}_{t-}, \mathbf{l} \neq \mathbf{k}\right\}}$ counting the transitions of $\mathbf{H}$ from $\mathbf{k}$ to $\mathbf{l}$, for every $\mathbf{k}, \mathbf{l} \in\{0,1\}^{n}$. Defining the measure $\mathbb{P}$ by $\frac{d \mathbb{P}}{d \mathbb{P}}=\Gamma_{T}$, it is then standard to check 10 that the point process $\mathbf{H}$ has intensity (3) under $\mathbb{P}$. To be precise the intensity of $N_{t}(\mathbf{k}, \mathbf{l})$ is given by (3), with respect to the model filtration $\mathcal{F}=\mathcal{F}^{(\mathbf{W}, \mathbf{H})}$, and the probability measure $\mathbb{P}$. Moreover, process $\mathbf{W}$ remains a Brownian motion under $\mathbb{P}$, the measure-change preserves Markov property of $\mathbf{X}$ with respect to filtration $\mathcal{F}$, and the generator of $\mathbf{X}$ under the new measure is still $A_{t}$.

Note that since martingale representation holds under $\widehat{\mathbb{P}} 11$ martingale representation also holds under the equivalent measure $\mathbb{P}$.

Remark A.1 The prevailing risk neutral probability measure in the paper is $\mathbb{P}$, whereas the auxiliary measure $\widehat{\mathbb{P}}$ is only a mathematical tool used for constructing the model, with no particular financial interpretation.

\section{A.1.1 Proof of Lemma 2.2}

By definition of the set-event indicator process $H^{Z}$, where $Z \in \mathcal{N}_{n}$, one has in our model, for $t \in[0, T]$,

$$
d H_{t}^{Z}=\sum_{\left\{\mathbf{k}, \mathbf{l} \in\{0,1\}^{n} ; \operatorname{supp}(\mathbf{l}) \backslash \operatorname{supp}(\mathbf{k})=Z\right\}} d N_{t}(\mathbf{k}, \mathbf{l}) .
$$

\footnotetext{
${ }^{9}$ Under suitable regularity and growth assumptions on the model coefficients, see Ethier and Kurtz [23] or Crépey [18.

${ }^{10}$ See for instance the proof of Lemma 8 in Crépey [18, or Lemma 7.5 in the online pre-print version of 18.

${ }^{11}$ In virtue of standard arguments, see for instance Chapter 10 of [30].
} 
So, by (3),

$$
\begin{aligned}
\ell_{t}^{Z} & =\sum_{\left\{\mathbf{k}, \mathbf{l} \in\{0,1\}^{n} ; \operatorname{supp}(\mathbf{l}) \backslash \operatorname{supp}(\mathbf{k})=Z\right\}} \sum_{\left\{\mathbf{l} \in\{0,1\}^{n} ; \operatorname{supp}(\mathbf{l}) \backslash \operatorname{supp}\left(\mathbf{H}_{t-}\right)=Z\right\}} \sum_{\left\{Y \in \mathcal{Y} ; \mathbf{H}_{t-}^{Y}=\mathbf{l}\right\}} \lambda_{\{Y \in} \sum_{\left\{Y \mathcal{Y} ; \mathbf{k}^{Y}=\mathbf{l}\right\}} \lambda_{Y}\left(t, X_{t}^{Y}\right) \\
= & \sum_{\left\{Y \in \mathcal{Y} ; \operatorname{supp}\left(\mathbf{H}_{t-}^{Y}\right) \backslash \operatorname{supp}\left(\mathbf{H}_{t-}\right)=Z\right\}} \lambda_{Y}\left(t, X_{t}^{Y}\right) \\
= & \sum_{\left\{Y \in \mathcal{Y} ; Y_{t}=Z\right\}} \lambda_{Y}\left(t, X_{t}^{Y}\right) .
\end{aligned}
$$

\section{A.1.2 Proof of Proposition 2.3}

Observe that $\left[M^{Y}, M^{Z}\right]=0$ for $Y \neq Z$. One thus has the following Itô formula (see for instance Theorem 3.89 page 109 of Jacod [29] or Crépey [18])

$$
\begin{aligned}
& d u\left(t, \mathbf{X}_{t}, \mathbf{H}_{t}\right)=\left(\partial_{t}+\mathcal{A}_{t}^{c}\right) u\left(t, \mathbf{X}_{t}, \mathbf{H}_{t}\right) d t+\nabla u\left(t, \mathbf{X}_{t}, \mathbf{H}_{t}\right) \sigma\left(t, \mathbf{X}_{t}\right) d \mathbf{W}_{t} \\
& +\sum_{Z \in \mathcal{N}_{n}} \delta u^{Z}\left(t, \mathbf{X}_{t}, \mathbf{H}_{t-}\right) d H_{t}^{Z}
\end{aligned}
$$

where

$$
\mathcal{A}_{t}^{c} u(t, \mathbf{x}, \mathbf{k})=\sum_{Y \in \mathcal{Y}}\left(b_{Y}\left(t, x_{Y}\right) \partial_{x_{Y}} u(t, \mathbf{x}, \mathbf{k})+\frac{1}{2} \sigma_{Y}^{2}\left(t, x_{Y}\right) \partial_{x_{Y}^{2}}^{2} u(t, \mathbf{x}, \mathbf{k})\right) .
$$

Moreover, the structure (44) of the set intensities implies that

$$
\sum_{Z \in \mathcal{N}_{n}} \delta u^{Z}\left(t, \mathbf{X}_{t}, \mathbf{H}_{t-}\right) d H_{t}^{Z}=\sum_{Z \in \mathcal{Z}_{t}} \delta u^{Z}\left(t, \mathbf{X}_{t}, \mathbf{H}_{t-}\right) d H_{t}^{Z},
$$

which we may further rewrite as

$$
\begin{aligned}
& \sum_{Z \in \mathcal{Z}_{t}} \ell_{Z}\left(t, \mathbf{X}_{t}, \mathbf{H}_{t-}\right) \delta u^{Z}\left(t, \mathbf{X}_{t}, \mathbf{H}_{t-}\right) d t \\
& \quad+\sum_{Z \in \mathcal{Z}_{t}}\left(\delta u^{Z}\left(t, \mathbf{X}_{t}, \mathbf{H}_{t-}\right) d H_{t}^{Z}-\ell_{Z}\left(t, \mathbf{X}_{t}, \mathbf{H}_{t}\right) \delta u^{Z}\left(t, \mathbf{X}_{t}, \mathbf{H}_{t}\right) d t\right)
\end{aligned}
$$

Here the second term is $\sum_{Z \in \mathcal{Z}_{t}} \delta u^{Z}\left(t, \mathbf{X}_{t}, \mathbf{H}_{t-}\right) d M_{t}^{Z}$, whereas one has by (44) in the first term:

$$
\begin{aligned}
\sum_{Z \in \mathcal{Z}_{t}} & \ell_{Z}\left(t, \mathbf{X}_{t}, \mathbf{H}_{t-}\right) \delta u^{Z}\left(t, \mathbf{X}_{t}, \mathbf{H}_{t-}\right) \\
= & \sum_{Z \in \mathcal{Z}_{t}} \sum_{Y \in \mathcal{Y} ; Y_{t}=Z} \lambda_{Y}\left(t, X_{t}^{Y}\right) \delta u^{Z}\left(t, \mathbf{X}_{t}, \mathbf{H}_{t-}\right) \\
= & \sum_{Y \in \mathcal{Y}} \lambda_{Y}\left(t, X_{t}^{Y}\right) \delta u^{Y}\left(t, \mathbf{X}_{t}, \mathbf{H}_{t-}\right)
\end{aligned}
$$

using in the last identity that

$$
\delta u^{Z}(t, \mathbf{x}, \mathbf{k})=\delta u^{Y}(t, \mathbf{x}, \mathbf{k})
$$

for every $t, \mathbf{x}, \mathbf{k}, Y$ and $Z$ such that $Y_{t}=Z$. Thus (54) indeed reduces to (6)). 


\section{A.2 Markov Properties}

Let us first recall the following local martingale characterization of a Markov process with generator $\mathcal{L}$. We work under the standing assumption that uniqueness holds for the solution of the martingale problem defined by $\mathcal{L}$.

Lemma A.2 (See, e.g., Ethier and Kurtz [23]) Let $X$ be a right-continuous process with Euclidean state space E, adapted to some filtration $\mathcal{F}$. For $X$ to be an $\mathcal{F}$ - Markov process with infinitesimal generator $\mathcal{L}$, it is necessary and sufficient that, for every realvalued function $\varphi$ in the domain of $\mathcal{L}$,

$$
\varphi\left(t, X_{t}\right)-\int_{0}^{t}\left(\partial_{s}+\mathcal{L}_{s}\right) \varphi\left(s, X_{s}\right) d s
$$

is an $\mathcal{F}$ - local martingale.

We shall use this characterization informally in this paper, ignoring the technicalities related to the notion of domain of an operator. Furthermore, throughout the paper we work under the standing assumption that the valuation equation associated to any infinitesimal generator that we use, is well posed in an appropriate functional space. Finally, we assume that uniqueness holds for the solution of the related martingale problem. The reader is referred to Ethier and Kurtz [23] for more details and for specific conditions which can be postulated in these regards.

\section{A.2.1 Proof of Proposition 2.9}

(i) In view of the Itô formula (6) $),(\mathbf{X}, \mathbf{H})$ solves the martingale problem with generator $\mathcal{A}$ in the filtration $\mathcal{F}$, and is thus an $\mathcal{F}$-Markov process.

(ii) By application of the local martingale characterization of an $\mathcal{F}$-Markov process $(\mathbf{X}, \mathbf{H})$ with generator $\mathcal{A}$ to test-functions of the form $u(t, \mathbf{x}, \mathbf{k})=v_{i}\left(t, x_{i}, k_{i}\right)$, we get the local martingale characterization of an $\mathcal{F}$ - Markov process with generator $\mathcal{A}^{i}$ for $\left(X^{i}, H^{i}\right)$. Considering $v_{i}\left(t, x_{i}, k_{i}\right)=\mathbb{1}_{k_{i}=1}$ therein yields that $M^{i}$ in (16) is an $\mathcal{F}$-local martingale.

(iii) We denote $t_{Z}=\max _{i \in Z} t_{i}$, for every $Z \in \mathcal{N}_{n}$. Formula (17) follows directly from Lemma A.3 below since one has, for every $t, t_{1}, \ldots, t_{n} \geq 0$,

$$
\begin{aligned}
\mathbb{P}\left(\tau_{1}>t_{1}, \ldots, \tau_{n}>t_{n} \mid \mathcal{F}_{t}\right) & =\sum_{Z \in \mathcal{N}_{n}} \mathbb{1}_{\left\{\operatorname{supp}^{c}\left(\mathbf{H}_{t}\right)=Z\right\}} \mathbb{P}\left(\tau_{1}>t_{1}, \ldots, \tau_{n}>t_{n} \mid \mathcal{F}_{t}\right) \\
& =\sum_{Z \in \mathcal{N}_{n}}\left(\prod_{i \notin Z} \mathbb{1}_{t_{i}<\tau_{i} \leq t}\right) \mathbb{E}\left\{\prod_{i \in Z} \mathbb{1}_{\tau_{i}>t_{i} \vee t} \mid \mathcal{F}_{t}\right\}
\end{aligned}
$$

and

$$
\begin{aligned}
\mathbb{1}_{\left\{t_{i}<\tau_{i}, i \in \operatorname{supp}\left(\mathbf{H}_{t}\right)\right\}} \mathbb{E}\left\{\exp \left(-\sum_{Y \in \mathcal{Y}} \Lambda_{t, \theta_{t}^{Y}}^{Y}\right) \mid \mathbf{X}_{t}\right\} \\
=\sum_{Z \in \mathcal{N}_{n}} \mathbb{1}_{\left\{\operatorname{supp}^{c}\left(\mathbf{H}_{t}\right)=Z\right\}} \mathbb{1}_{\left\{t_{i}<\tau_{i}, i \notin Z\right\}} \mathbb{E}\left\{\exp \left(-\sum_{Y \in \mathcal{Y}} \Lambda_{t, \theta_{t}^{Y}}^{Y}\right) \mid \mathbf{X}_{t}\right\} \\
=\sum_{Z \in \mathcal{N}_{n}}\left(\prod_{i \notin Z} \mathbb{1}_{t_{i}<\tau_{i} \leq t}\right)\left(\prod_{i \in Z} \mathbb{1}_{\tau_{i}>t}\right) \mathbb{E}\left\{\exp \left(-\sum_{Y \in \mathcal{Y}} \Lambda_{t, t_{Y \cap Z}}^{Y}\right) \mid \mathbf{X}_{t}\right\} .
\end{aligned}
$$

Given (17), the other formulas of part (iii) in Proposition 2.9 are straightforward. 
Lemma A.3 For every $t, t_{1}, \ldots, t_{n} \geq 0$, and for every $Z \in \mathcal{N}_{n}$, one has,

$$
\mathbb{E}\left\{\prod_{i \in Z} \mathbb{1}_{\tau_{i}>t_{i} \vee t} \mid \mathcal{F}_{t}\right\}=\left(\prod_{i \in Z} \mathbb{1}_{\tau_{i}>t}\right) \mathbb{E}\left\{\exp \left(-\sum_{Y \in \mathcal{Y} ; Y \cap Z \neq \emptyset} \Lambda_{t, t_{Y}}^{Y}\right) \mid \mathbf{X}_{t}\right\}
$$

Proof. It is enough to prove that for $t_{i} \geq t$ one has, for every $Z \in \mathcal{N}_{n}$,

$$
\mathbb{E}\left\{\prod_{i \in Z} \mathbb{1}_{\tau_{i}>t_{i}} \mid \mathcal{F}_{t}\right\}=\left(\prod_{i \in Z} \mathbb{1}_{\tau_{i}>t}\right) \mathbb{E}\left\{\exp \left(-\sum_{Y \in \mathcal{Y}} \Lambda_{t, t_{Y \cap Z}}^{Y}\right) \mid \mathbf{X}_{t}\right\} .
$$

Indeed, for general $t_{i}$, applying (58) to the $t_{i} \vee t$ yields

$$
\begin{gathered}
\mathbb{E}\left\{\prod_{i \in Z} \mathbb{1}_{\tau_{i}>t_{i} \vee t} \mid \mathcal{F}_{t}\right\}=\left(\prod_{i \in Z} \mathbb{1}_{\tau_{i}>t}\right) \mathbb{E}\left\{\exp \left(-\sum_{Y \in \mathcal{Y}} \Lambda_{t, \max _{i \in Y \cap Z} t_{i} \vee t}\right) \mid \mathbf{X}_{t}\right\} \\
=\left(\prod_{i \in Z} \mathbb{1}_{\tau_{i}>t}\right) \mathbb{E}\left\{\exp \left(-\sum_{Y \in \mathcal{Y} ; Y \cap Z \neq \emptyset} \Lambda_{t, \max _{i \in Z} t_{i}}^{Y} \mid \mathbf{X}_{t}\right\}\right.
\end{gathered}
$$

which is (57). Let us thus show (58) for $t_{i} \geq t$, by induction on the cardinality $d$ of $Z$. For $d=0$, the result is trivial. Assuming the result at rank $d-1 \geq 0$, let us show the result at rank $d$. Let us suppose, without loss of generality, that $Z=\mathbb{N}_{d}$ and $t_{1} \geq t_{2} \geq \cdots \geq t_{d} \geq t$. One then needs to prove that, using the notation $J^{l}=1-H^{l}$ for every $l \in \mathbb{N}_{d}$,

$$
\mathbb{E}\left(\prod_{l=1}^{d} J_{t_{l}}^{l} \mid \mathcal{F}_{t}\right)=\left(\prod_{l=1}^{d} J_{t}^{l}\right) \mathbb{E}\left\{\exp \left(-\sum_{Y \in \mathcal{Y}} \Lambda_{t, t_{\mathbb{N}_{d}} \cap Y}\right) \mid \mathbf{X}_{t}\right\} .
$$

To establish (59) one first observes that

$$
\mathbb{E}\left(\prod_{l=1}^{d} J_{t_{l}}^{l} \mid \mathcal{F}_{t}\right)=\mathbb{E}\left\{J_{t_{d}}^{d} \mathbb{E}\left\{\prod_{l=1}^{d-1} J_{t_{l}}^{l} \mid \mathcal{F}_{t_{d}}\right\} \mid \mathcal{F}_{t}\right\}
$$

where by the induction hypothesis at rank $d-1$ the inner conditional expectation can be represented as

$$
\left(\prod_{l=1}^{d-1} J_{t_{d}}^{l}\right) \mathbb{E}\left\{\exp \left(-\sum_{Y \in \mathcal{Y}} \Lambda_{t_{d}, t_{\mathbb{N}_{d}-1} \cap Y}^{Y}\right) \mid \mathbf{X}_{t_{d}}\right\}=\left(\prod_{l=1}^{d-1} J_{t_{d}}^{l}\right) v\left(t_{d}, \mathbf{X}_{t_{d}}\right)
$$

for a suitable function $v=v(t, \mathbf{x})$ over $\left[0, t_{d-1}\right] \times \mathbb{R}^{\mathcal{Y}}$, by the Markov property of $\mathbf{X}$. Here the upper bound $t_{d-1}$ for the domain of definition of the function $v$ follows from the fact that $t_{d} \leq t_{d-1} \leq t_{\mathbb{N}_{d-1} \cap Y}$, for every $Y \in \mathcal{Y}$ with $\mathbb{N}_{d-1} \cap Y \neq \emptyset$. Inserting (61) into (60) yields by the Markov property of $(\mathbf{X}, \mathbf{H})$ that

$$
\mathbb{E}\left(\prod_{l=1}^{d} J_{t_{l}}^{l} \mid \mathcal{F}_{t}\right)=\mathbb{E}\left\{\left(\prod_{l=1}^{d} J_{t_{d}}^{l}\right) v\left(t_{d}, \mathbf{X}_{t_{d}}\right) \mid \mathcal{F}_{t}\right\}=u\left(t, \mathbf{X}_{t}, \mathbf{H}_{t}\right),
$$

for a function $u=u(t, \mathbf{x}, \mathbf{k})$ over $\left[0, t_{d}\right] \times \mathbb{R}^{\mathcal{Y}} \times\{0,1\}^{n}$ characterized by:

$$
\left\{\begin{array}{l}
u\left(t_{d}, \mathbf{x}, \mathbf{k}\right)=\left(\prod_{l=1}^{d}\left(1-k_{l}\right)\right) v\left(t_{d}, \mathbf{x}\right), \quad \mathbf{x}=\left(x_{Y}\right)_{Y \in \mathcal{Y}}, \mathbf{k}=\left(k_{1}, \ldots, k_{n}\right) \in\{0,1\}^{n} \\
\left(\partial_{t}+\mathcal{A}_{t}\right) u(t, \mathbf{x}, \mathbf{k})=0, \quad t<t_{d}, \mathbf{x}=\left(x_{Y}\right)_{Y \in \mathcal{Y}}, \mathbf{k} \in\{0,1\}^{n}
\end{array}\right.
$$


One finally shows that the RHS in (59) admits a representation of the form $\left(\prod_{l=1}^{d} J_{t}^{l}\right) w\left(t, \mathbf{X}_{t}\right)$, where the function $\tilde{u}(t, \mathbf{x}, \mathbf{k})=\left(\prod_{l=1}^{d}\left(1-k_{l}\right)\right) w(t, \mathbf{x})$ solves (62). By our standing assumption in this paper equation (62) has a unique solution. Thus $\tilde{u}=u$, which proves (59).

Since $\mathbf{X}$ is Markov with generator $A$ (cf. (i)), the conditional expectation in the RHS of (59) can be represented as $w\left(t, \mathbf{X}_{t}\right)$, for a deterministic function $w=w(t, \mathbf{x})$ over the domain $\left[0, t_{d}\right] \times \mathbb{R}^{\mathcal{Y}}$. In order to get the analytic characterization of $w$, first note that for every $Y \in \mathcal{Y}$, one has:

$$
\Lambda_{t_{d}, t_{\mathbb{N}_{d-1}} \cap Y}^{Y}=\Lambda_{t_{d}, t_{\mathbb{N}_{d} \cap Y}}^{Y}=\Lambda_{t_{d}, t_{\mathbb{N}_{d}} \cap Y}^{Y}
$$

This yields the terminal condition $w\left(t_{d}, \mathbf{x}\right)=v\left(t_{d}, \mathbf{x}\right), \mathbf{x}=\left(x_{Y}\right)_{Y \in \mathcal{Y}}$. One further has by an application of the Feynman-Kac formula that (see, e.g., Jeanblanc et al. [30])

$$
\left(\partial_{t}+A_{t}\right) w(t, \mathbf{x})=\left(\sum_{Y \in \mathcal{Y} ; Y \cap Z \neq \emptyset} \lambda_{Y}\left(t, x_{Y}\right)\right) w(t, \mathbf{x}), \quad t<t_{d}, \mathbf{x}=\left(x_{Y}\right)_{Y \in \mathcal{Y}} .
$$

As a result the function $w=w(t, \mathbf{x})$ is the solution on $\left[0, t_{d}\right] \times \mathbb{R}^{\mathcal{Y}}$ to the following Kolmogorov pricing PDE:

$$
\left\{\begin{array}{l}
w\left(t_{d}, \mathbf{x}\right)=v\left(t_{d}, \mathbf{x}\right), \quad \mathbf{x}=\left(x_{Y}\right)_{Y \in \mathcal{Y}} \\
\left(\partial_{t}+A_{t}\right) w(t, \mathbf{x})=\left(\sum_{Y \in \mathcal{Y} ; Y \cap Z \neq \emptyset} \lambda_{Y}\left(t, x_{Y}\right)\right) w(t, \mathbf{x}), \quad t<t_{d}, \mathbf{x}=\left(x_{Y}\right)_{Y \in \mathcal{Y}} .
\end{array}\right.
$$

Denoting $\tilde{u}(t, \mathbf{x}, \mathbf{k})=\left(\prod_{l \in \mathbb{N}_{d}}\left(1-k_{l}\right)\right) w(t, \mathbf{x})$, an application of the operator $\mathcal{A}_{t}$ of (7) yields:

$$
\begin{aligned}
\left(\partial_{t}+\mathcal{A}_{t}\right) \tilde{u}(t, \mathbf{x}, \mathbf{k})= & \left(\prod_{l \in \mathbb{N}_{d}}\left(1-k_{l}\right)\right)\left(\partial_{t}+A_{t}\right) w(t, \mathbf{x})+w(t, \mathbf{x}) \times \\
& \times \sum_{Y \in \mathcal{Y}} \lambda_{Y}\left(t, x_{Y}\right)\left(\left(\prod_{l \in \mathbb{N}_{d}}\left(1-k_{l}^{Y}\right)\right)-\prod_{l \in \mathbb{N}_{d}}\left(1-k_{l}\right)\right),
\end{aligned}
$$

where we set, for $Y \in \mathcal{Y}$ and $l \in \mathbb{N}_{d}$,

$$
k_{l}^{Y}= \begin{cases}1, & Y \ni l \\ k_{l}, & \text { else. }\end{cases}
$$

Therefore

$$
\begin{aligned}
\sum_{Y \in \mathcal{Y}} \lambda_{Y}\left(t, x_{Y}\right)\left(\left(\prod_{l \in \mathbb{N}_{d}}\left(1-k_{l}^{Y}\right)\right)-\prod_{l \in \mathbb{N}_{d}}\left(1-k_{l}\right)\right) \\
=-\prod_{l \in \mathbb{N}_{d}}\left(1-k_{l}\right) \sum_{Y \in \mathcal{Y} ; Y \cap \mathbb{N}_{d} \neq \emptyset} \lambda_{Y}\left(t, x_{Y}\right) .
\end{aligned}
$$

Plugging (63) and (65) in the RHS of (64) yields that $\left(\partial_{t}+\mathcal{A}_{t}\right) \tilde{u}(t, \mathbf{x}, \mathbf{k})=0$. Finally $\tilde{u}$ solves (62), which finishes the demonstration.

\section{A.2.2 Proof of Proposition 3.1}

Given a function $f=f(t, y)$, let $f\left(t_{j}-, x\right)$ be a notation for the formal limit

$$
\lim _{(t, y) \rightarrow\left(t_{j}, x\right) \text { with } t<t_{j}} f(t, y) .
$$

In view of the Markov properties of the model gathered in Proposition 2.9, the following lemma holds in virtue of the Feynman-Kac formula 12

\footnotetext{
${ }^{12}$ See, e.g., Jeanblanc et al. [30].
} 
Lemma A.4 (i) Given real numbers $\phi_{i}$ and $\psi_{i}$, one has $\mathbb{E}\left[\phi_{i} \sum_{t<t_{j} \leq T}\left(1-H_{t_{j}}^{i}\right)+\psi_{i} H_{T}^{i} \mid \mathcal{F}_{t}\right]=$ $\left(1-H_{t}^{i}\right) v_{i}\left(t, X_{t}^{i}\right)+\psi_{i} H_{t}^{i}$, where the pre-default function $v_{i}\left(t, x_{i}\right)$ solves the following Kolmogorov equation: $v_{i}\left(T, x_{i}\right)=0, x_{i} \in \mathbb{R}$, and for $j$ decreasing from $p$ to 1 :

- At $t=t_{j}$

$$
v_{i}\left(t_{j}-, x_{i}\right)=v_{i}\left(t_{j}, x_{i}\right)+\phi_{i}, x_{i} \in \mathbb{R},
$$

- On the time interval $\left[t_{j-1}, t_{j}\right)$,

$$
\left(\partial_{t}+A_{t}^{i}\right) v_{i}\left(t, x_{i}\right)+\lambda_{i}\left(t, x_{i}\right) \psi_{i}=0, x_{i} \in \mathbb{R} .
$$

(ii) Given real-valued functions $\phi(\mathbf{k})$ and $\psi(\mathbf{k})$, one has $\mathbb{E}\left[\sum_{t<t_{j} \leq T} \phi\left(\mathbf{H}_{t_{j}}\right)+\psi\left(\mathbf{H}_{T}\right) \mid \mathcal{F}_{t}\right]=$ $w\left(t, \mathbf{X}_{t}, \mathbf{H}_{t}\right)$, where the function $w(t, \mathbf{x}, \mathbf{k})$ is the solution to the following Kolmogorov pricing PDE system: $w(T, \mathbf{x}, \mathbf{k})=\psi(\mathbf{k}), \mathbf{x}=\left(x_{Y}\right)_{Y \in \mathcal{Y}}, \mathbf{k} \in\{0,1\}^{n}$, and for $j$ decreasing from $p$ to 1:

- At $t=t_{j}$,

$$
w\left(t_{j}-, \mathbf{x}, \mathbf{k}\right)=w\left(t_{j}, \mathbf{x}, \mathbf{k}\right)+\phi(\mathbf{k}), \mathbf{x}=\left(x_{Y}\right)_{Y \in \mathcal{Y}}, \mathbf{k} \in\{0,1\}^{n},
$$

- On the time interval $\left[t_{j-1}, t_{j}\right)$,

$$
\left(\partial_{t}+\mathcal{A}_{t}\right) w(t, \mathbf{x}, \mathbf{k})=0, \mathbf{x}=\left(x_{Y}\right)_{Y \in \mathcal{Y}}, \mathbf{k} \in\{0,1\}^{n} .
$$

Applying this Lemma with

$$
\psi_{i}=\left(1-R_{i}\right), \phi_{i}=-S_{i} \mathrm{~h}
$$

in part (i) and

$$
\psi=L_{a, b}, \quad \phi=-S \mathrm{~h}\left(b-a-L_{a, b}\right)
$$

in part (ii), establishes the first lines in identities (24) and (25). Regarding the latter, note that the ex-dividend pricing function $u(t, \mathbf{k}, \mathbf{x})$ in (25), is provided by $w(t, \mathbf{k}, \mathbf{x})-L_{a, b}(\mathbf{k})$ here.

Moreover, in the filtration $\mathcal{F}=\mathcal{F}^{\mathbf{W}, \mathbf{H}}$, a martingale can only jump at totally unpredictable stopping times. In particular, the cumulative value processes cannot jump at the fixed times $t_{j}$. Given the first lines in (24) and (25), the second lines then readily follow using the Itô formula ([6).

\section{References}

[1] Andersen, L. And Sidenius, J.: Extensions to the Gaussian Copula: Random Recovery and Random Factor Loadings, Journal of Credit Risk, Vol. 1, No. 1 (Winter 2004), p. 29-70.

[2] Assefa, S., Bielecki, T.R., Crépey, S. and Jeanblanc, M.: CVA computation for counterparty risk assessment in credit portfolios. Credit Risk Frontiers, Bielecki, T.R., Brigo, D. and Patras, F., eds., Wiley/Bloomberg-Press, 2011.

[3] Basel Committee on Banking Supervision: Revisions to the Basel II market risk framework, December 2010. 
[4] Bielecki, T.R., Crépey, S. and Jeanblanc, M.: Up and Down Credit Risk.Quantitative Finance, p. 1-15, April 2010.

[5] Bielecki, T.R., Cousin, A., Crépey, S., Herbertsson, A.: A Markov Copula Model of Portfolio Credit Risk with Stochastic Intensities and Random Recoveries. Submitted 2012.

[6] Bielecki, T. R. And Crépey, S.: Dynamic Hedging of Counterparty Exposure. Forthcoming in The Musiela Festschrift, Zariphopoulou, T., Rutkowski, M. and Kabanov, Y., eds, Springer.

[7] Bielecki, T.R., Crépey, S., Jeanblanc, M. and Zargari, B.: Valuation and Hedging of CDS Counterparty Exposure in a Markov Copula Model. Forthcoming in International Journal of Theoretical and Applied Finance.

[8] Bielecki, T. R. and Jakubowski, J. and Niewȩglowski, M.: Dynamic Modeling of Dependence in Finance via Copulae Between Stochastic Processes, Copula Theory and Its Applications, Lecture Notes in Statistics, Vol.198, Part 1, 33-76, 2010.

[9] Bielecki, T.R. And Rutkowski, M.: Credit Risk: Modeling, Valuation and Hedging. Springer-Verlag, Berlin, 2002.

[10] Bielecki, T.R., Cialenco, I. and Iyigunler, I.: Collateralized CVA Valuation with Rating Triggers and Credit Migrations, submitted, 2012.

[11] Bielecki, T.R., Vidozzi, A. And Vidozzi, L.: A Markov Copulae Approach to Pricing and Hedging of Credit Index Derivatives and Ratings Triggered Step-Up Bonds, J. of Credit Risk, 2008.

[12] Brigo, D., Pallavicini, A., Torresetti, R.: Calibration of CDO Tranches with the dynamical Generalized-Poisson Loss model. Risk Magazine, May 2007

[13] Brigo, D., Pallavicini, A., Torresetti, R. Credit models and the crisis: default cluster dynamics and the generalized Poisson loss model, Journal of Credit Risk, 6(4), 39-81, 2010.

[14] Brigo, D., Pallavicini, A., Torresetti, R. Cluster-based extension of the generalized poisson loss dynamics and consistency with single names. International Journal of Theoretical and Applied Finance, Vol 10, n. 4, 607-632, 2007.

[15] Cont, R. And Kan, Y.H.: Dynamic Hedging of Portfolio Credit Derivatives, SIAM Journal on Financial Mathematics, 2, p. 112-140, 2011.

[16] Cont, R., Minca, A.: Recovering Portfolio Default Intensities Implied by CDO Quotes, Working Paper, 2008.

[17] Cousin, A., Crépey, S. and Kan, Y.H.: Delta-hedging Correlation Risk? Review of Derivatives Research, June 2011.

[18] Crépey, S.: About the pricing equations in finance. Paris-Princeton Lectures in Mathematical Finance 2010, Lecture Notes in Mathematics, Springer, p.63-203, 2011.

[19] Crépey, S. and Rahal, A.: Simulation/Regression Pricing Schemes for CVA Computations on CDO Tranches. Working Paper, 2012. 
[20] Cont, R., Deguest, R. and Kan, Y. H.: Recovering Default Intensity from CDO Spreads: Inversion Formula and Model Calibration, SIAM Journal on Financial Mathematics, 1, p. 555-585, 2010.

[21] Duffie, D. and Gârleanu, N.: Risk and the valuation of collateralized debt obligations, Financial Analysts Journal, 57, 41-62, 2001.

[22] Elouerkhaoui, Y.: Pricing and Hedging in a Dynamic Credit Model. International Journal of Theoretical and Applied Finance, Vol. 10, Issue 4, 703-731, 2007.

[23] Ethier, H.J. And Kurtz, T.G.: Markov Processes. Characterization and Convergence. Wiley, 1986.

[24] Frey, R., Backhaus, J. Dynamic hedging of synthetic CDO tranches with spreadand contagion risk, Journal of Economic Dynamics and Control, 34(4), 710-724, 2010.

[25] Giesecke, K., Goldberg, L.: A Top Down Approach to Multi-Name Credit. Working Paper, 2007.

[26] Herbertsson, A. (2008). Pricing synthetic CDO tranches in a model with Default Contagion using the Matrix-Analytic approach, The Journal of Credit Risk 4(4), 3-35.

[27] Herbertsson, A. (2011) Modelling default contagion using Multivariate Phase-Type distributions, Review of Derivatives Research 14(1), 1-36.

[28] Herbertsson, A. And Rootzén, H. (2008) Pricing kth-to-default swaps under default contagion:the matrix-analytic approach, The Journal of Computational Finance $12(1), 49-78$.

[29] Jacod, J.: Calcul Stochastique et Problèmes de Martingales. Springer, 2nd edition, 2003.

[30] Jeanblanc, M., Yor, M. and Chesney, M.: Mathematical methods for Financial Markets. Springer, 2009.

[31] Laurent, J.-P., Cousin, A. and Fermanian, J-D.: Hedging default risks of CDOs in Markovian contagion models. Quantitative Finance, 1469-7696, April 2010.

[32] Iscoe, I., Jackson, K., Kreinin, A. And Ma, X.: On Exponential Approximation to the Hockey Stick Function. Working Paper, Department of Computer Science, University of Toronto, 2010.

[33] Iscoe, I., Jackson, K., Kreinin, A. And Ma, X.: Pricing correlation-dependent derivatives based on exponential approximations to the hockey stick function. Forthcoming in Journal of Computational Finance.

[34] Beylkin, G. And Monzon, L. On approximation of functions by exponential sums, Applied and Computational Harmonic Analysis, 19(1): 17-48, 2005.

[35] Lindskog, F. And McNeil, A. J.: Common Poisson shock models: applications to insurance and credit risk modelling. ASTIN Bulletin, 33(2), 209-238, 2003

[36] Marshall, A. \& Olkin, I.: A multivariate exponential distribution, J. Amer. Statist. Assoc., 2, 84-98, 1967. 
[37] Patton, A.: Modelling Time-varying exchange rate dependence using the conditional copula, Working Paper 2001-09, University of California, San Diego, 2001.

[38] Protter, P.E.: Stochastic Integration and Differential Equations, Second Edition, Version 2.1. Springer, 2005.

[39] Rogers, L.C.G. And Pitman J.W.: Markov functions, Ann. of Prob., 9, 578-582, 1981.

[40] Schweizer, M.: A Guided Tour through Quadratic Hedging Approaches. In Option Pricing, Interest Rates and Risk Management, E. Jouini, J. Cvitanic and M. Musiela, eds., Cambridge University Press, 538-574, 2001. 